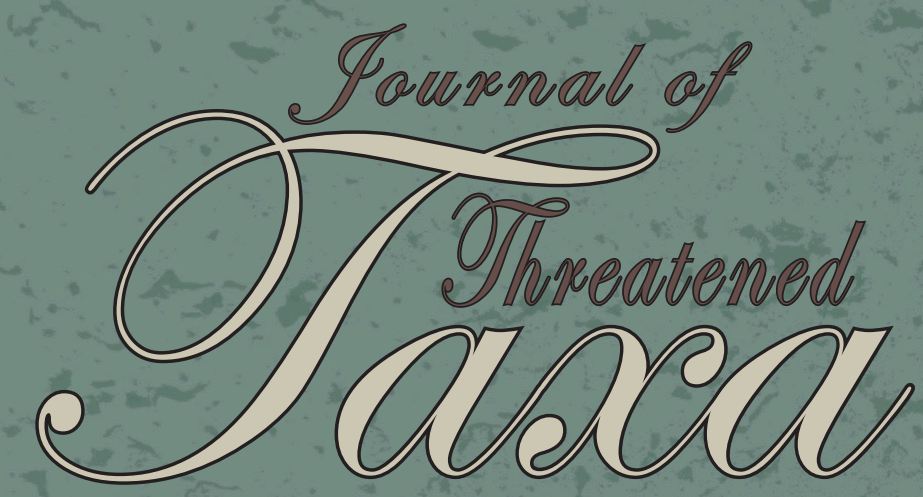

Building evidence for conservation globally
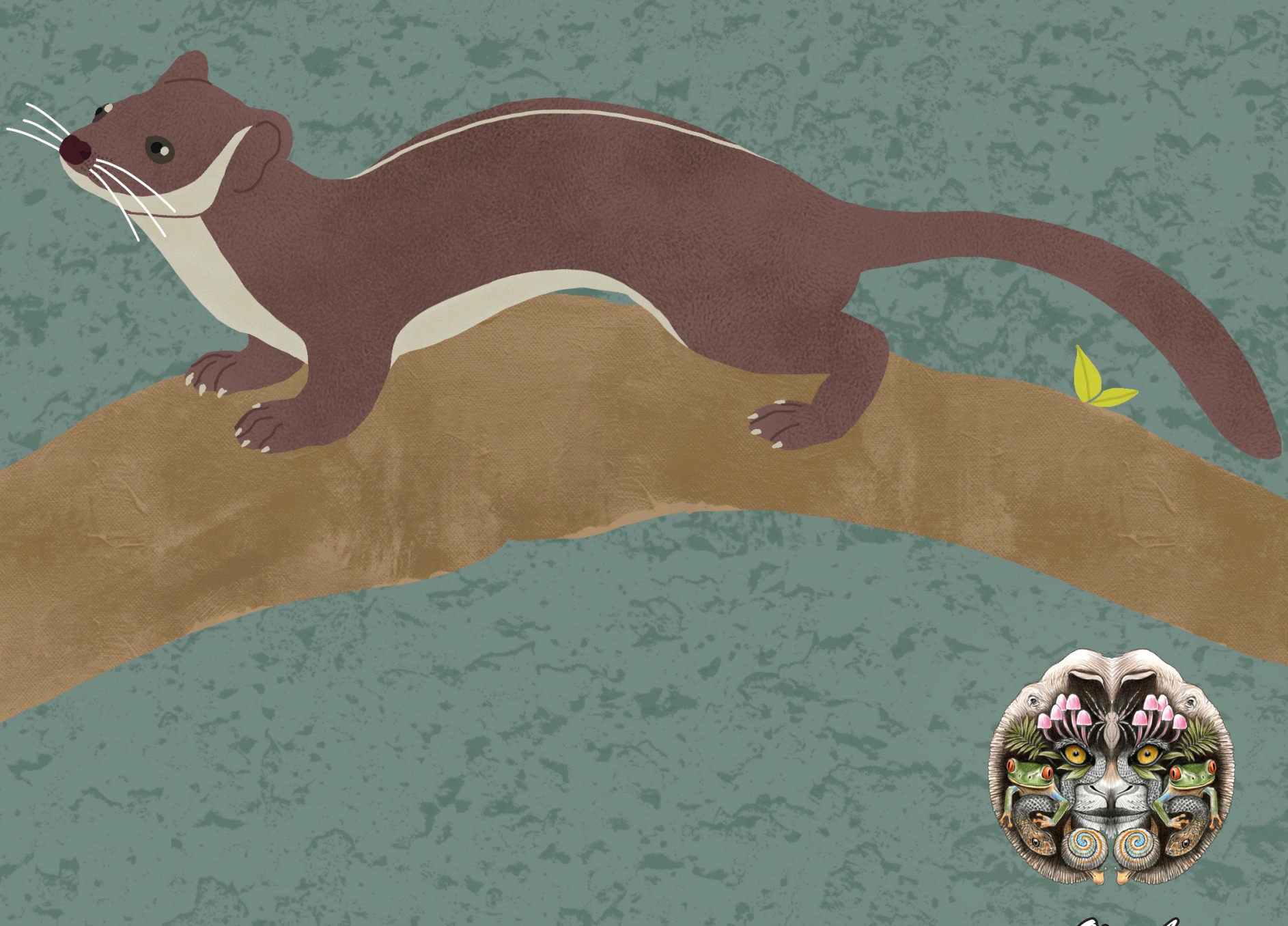

Qpecosecess

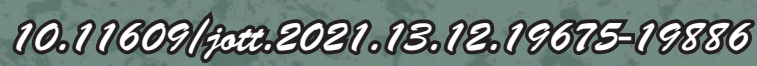
cocosurthreatecredtassararg

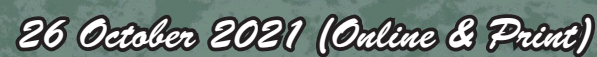

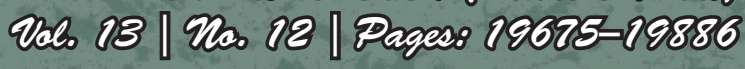






ISSN 0974-7907 (Online); ISSN $0974-7893$ (Print)

Publisher

Host

Wildlife Information Liaison Development Society

www.wild.zooreach.org

Zoo Outreach Organization www.zooreach.org

No. 12, Thiruvannamalai Nagar, Saravanampatti - Kalapatti Road, Saravanampatti, Coimbatore, Tamil Nadu 641035, India

Ph: +91 9385339863 | www.threatenedtaxa.org

Email: sanjay@threatenedtaxa.org

EDITORS

\section{Founder \& Chief Editor}

Dr. Sanjay Molur

Wildlife Information Liaison Development (WILD) Society \& Zoo Outreach Organization (ZOO)

12 Thiruvannamalai Nagar, Saravanampatti, Coimbatore, Tamil Nadu 641035, India

\section{Deputy Chief Edito}

Dr. Neelesh Dahanukar

Noida, Uttar Pradesh, India

\section{Managing Editor}

Mr. B. Ravichandran, WILD/ZOO, Coimbatore, India

\section{Associate Editors}

Dr. Mandar Paingankar, Government Science College Gadchiroli, Maharashtra 442605, India Dr. Ulrike Streicher, Wildlife Veterinarian, Eugene, Oregon, USA

Ms. Priyanka Iyer, ZOO/WILD, Coimbatore, Tamil Nadu 641035, India

Dr. B. A. Daniel, $200 /$ WIID, Coimbatore, Tamil Nadu 641035, India

\section{Editorial Board}

Dr. Russel Mittermeie

Executive Vice Chair, Conservation International, Arlington, Virginia 22202, USA

\section{Prof. Mewa Singh Ph.D., FASc, FNA, FNASc, FNAPsy}

Ramanna Fellow and Life-Long Distinguished Professor, Biopsychology Laboratory, and Institute of Excellence, University of Mysore, Mysuru, Karnataka 570006, India; Honorary Professor, Jawaharlal Nehru Centre for Advanced Scientific Research, Bangalore; and Adjunct Professor, National Institute of Advanced Studies, Bangalore

\section{Stephen D. Nash}

Scientific Illustrator, Conservation International, Dept. of Anatomical Sciences, Health Sciences Center, T-8, Room 045, Stony Brook University, Stony Brook, NY 11794-8081, USA

\section{Dr. Fred Pluthero}

Toronto, Canada

\section{Dr. Priya Davidar}

Sigur Nature Trust, Chadapatti, Mavinhalla PO, Nilgiris, Tamil Nadu 643223, India

\section{Dr. Martin Fisher}

Senior Associate Professor, Battcock Centre for Experimental Astrophysics, Cavendish

Laboratory, JJ Thomson Avenue, Cambridge CB3 OHE, UK

\section{Dr. John Fellowes}

Honorary Assistant Professor, The Kadoorie Institute, 8/F, T.T. Tsui Building, The University of Hong Kong, Pokfulam Road, Hong Kong

\section{Prof. Dr. Mirco Solé}

Universidade Estadual de Santa Cruz, Departamento de Ciências Biológicas, Vice-coordenado do Programa de Pós-Graduação em Zoologia, Rodovia Ilhéus/Itabuna, Km 16 (45662-000)

Salobrinho, Ilhéus - Bahia - Brasil

\section{Dr. Rajeev Raghavan}

Professor of Taxonomy, Kerala University of Fisheries \& Ocean Studies, Kochi, Kerala, India

\section{English Editors}

Mrs. Mira Bhojwani, Pune, India

Dr. Fred Pluthero, Toronto, Canad

Mr. P. Ilangovan, Chennai, India

Web Maintenance

Mrs. Latha G. Ravikumar, ZOO/WILD, Coimbatore, India

\section{Typesetting}

Mr. Arul Jagadish, ZOO, Coimbatore, India

Mrs. Radhika, ZOO, Coimbatore, India

Mrs. Geetha, ZOO, Coimbatore India

\section{Fundraising/Communications}

Mrs. Payal B. Molur, Coimbatore, India

Subject Editors 2018-2020

Fungi

Dr. B. Shivaraju, Bengaluru, Karnataka, India

Dr. R.K. Verma, Tropical Forest Research Institute, Jabalpur, India

Dr. Vatsavaya S. Raju, Kakatiay University, Warangal, Andhra Pradesh, India

Dr. M. Krishnappa, Jnana Sahyadri, Kuvempu University, Shimoga, Karnataka, India

Dr. K.R. Sridhar, Mangalore University, Mangalagangotri, Mangalore, Karnataka, India

Dr. Gunjan Biswas, Vidyasagar University, Midnapore, West Bengal, India

Plants

Dr. G.P. Sinha, Botanical Survey of India, Allahabad, India

Dr. N.P. Balakrishnan, Ret. Joint Director, BSI, Coimbatore, India

Dr. Shonil Bhagwat, Open University and University of Oxford, UK

Prof. D.J. Bhat, Retd. Professor, Goa University, Goa, India

Dr. Ferdinando Boero, Università del Salento, Lecce, Italy

Dr. Dale R. Calder, Royal Ontaro Museum, Toronto, Ontario, Canada

Dr. Cleofas Cervancia, Univ. of Philippines Los Baños College Laguna, Philippines

Dr. F.B. Vincent Florens, University of Mauritius, Mauritius

Dr. Merlin Franco, Curtin University, Malaysia

Dr. V. Irudayaraj, St. Xavier's College, Palayamkottai, Tamil Nadu, India

Dr. B.S. Kholia, Botanical Survey of India, Gangtok, Sikkim, India

Dr. Pankaj Kumar, Kadoorie Farm and Botanic Garden Corporation, Hong Kong S.A.R., China

Dr. V. Sampath Kumar, Botanical Survey of India, Howrah, West Bengal, India

Dr. A.J. Solomon Raju, Andhra University, Visakhapatnam, India

Dr. Vijayasankar Raman, University of Mississippi, USA

Dr. B. Ravi Prasad Rao, Sri Krishnadevaraya University, Anantpur, India

Dr. K. Ravikumar, FRLHT, Bengaluru, Karnataka, India

Dr. Aparna Watve, Pune, Maharashtra, India

Dr. Qiang Liu, Xishuangbanna Tropical Botanical Garden, Yunnan, China

Dr. Noor Azhar Mohamed Shazili, Universiti Malaysia Terengganu, Kuala Terengganu, Malaysia Dr. M.K. Vasudeva Rao, Shiv Ranjani Housing Society, Pune, Maharashtra, India

Prof. A.J. Solomon Raju, Andhra University, Visakhapatnam, India

Dr. Mandar Datar, Agharkar Research Institute, Pune, Maharashtra, India

Dr. M.K. Janarthanam, Goa University, Goa, India

Dr. K. Karthigeyan, Botanical Survey of India, India

Dr. Errol Vela, University of Montpellier, Montpellier, France

Dr. P. Lakshminarasimhan, Botanical Survey of India, Howrah, India

Dr. Larry R. Noblick, Montgomery Botanical Center, Miami, USA

Dr. K. Haridasan, Pallavur, Palakkad District, Kerala, India

Dr. Analinda Manila-Fajard, University of the Philippines Los Banos, Laguna, Philippines

Dr. P.A. Sinu, Central University of Kerala, Kasaragod, Kerala, India

Dr. Afroz Alam, Banasthali Vidyapith (accredited A grade by NAAC), Rajasthan, India

Dr. K.P. Rajesh, Zamorin's Guruvayurappan College, GA College PO, Kozhikode, Kerala, India

Dr. David E. Boufford, Harvard University Herbaria, Cambridge, MA 02138-2020, USA

Dr. Ritesh Kumar Choudhary, Agharkar Research Institute, Pune, Maharashtra, India

Dr. Navendu Page, Wildlife Institute of India, Chandrabani, Dehradun, Uttarakhand, India

Invertebrates

Dr. R.K. Avasthi, Rohtak University, Haryana, India

Dr. D.B. Bastawade, Maharashtra, India

Dr. Partha Pratim Bhattacharjee, Tripura University, Suryamaninagar, India

Dr. Kailash Chandra, Zoological Survey of India, Jabalpur, Madhya Pradesh, India

Dr. Ansie Dippenaar-Schoeman, University of Pretoria, Queenswood, South Africa

Dr. Rory Dow, National Museum of natural History Naturalis, The Netherlands

Dr. Brian Fisher, California Academy of Sciences, USA

Dr. Richard Gallon, llandudno, North Wales, LL30 1UP

Dr. Hemant V. Ghate, Modern College, Pune, India

Dr. M. Monwar Hossain, Jahangirnagar University, Dhaka, Bangladesh

Mr. Jatishwor Singh Irungbam, Biology Centre CAS, Branišovská, Czech Republic.

Dr. lan J. Kitching, Natural History Museum, Cromwell Road, UK

Dr. George Mathew, Kerala Forest Research Institute, Peechi, India

Dr. John Noyes, Natural History Museum, London, UK

For Focus, Scope, Aims, and Policies, visit https://threatenedtaxa.org/index.php/JoTT/aims_scope
For Article Submission Guidelines, visit https://threatenedtaxa.org/index.php/JoTT/about/submissions
For Policies against Scientific Misconduct, visit https://threatenedtaxa.org/index.php/JoTT/policies_various

continued on the back inside cover 


\title{
Diversity and distribution of avifauna at Warathenna-Hakkinda Environmental Protection Area in Kandy, Sri Lanka
}

\section{Dinelka Thilakarathne ${ }^{1}$ (D), Tithira Lakkana ${ }^{2}$ (D), Gayan Hirimuthugoda ${ }^{3}$ (D), Chaminda Wijesundara ${ }^{4}$ (D) \& Shalika Kumburegama ${ }^{5}$ (D)}

\author{
1,3,4,5 Department of Zoology, Faculty of Science, University of Peradeniya, Peradeniya, Sri Lanka. \\ ${ }^{1}$ Postgraduate Institute of Science, University of Peradeniya, Peradeniya, Sri Lanka. \\ ${ }^{2}$ Faculty of Science and Technology, Uva Wellassa University, Passara Road, Badulla, Sri Lanka. \\ ${ }^{1}$ dinelkat@yahoo.com, ${ }^{2}$ tithiralakkana@gmail.com, ${ }^{3}$ nadeela@lankamail.com, ${ }^{4}$ chamindaw@sci.pdn.ac.lk, \\ ${ }^{5}$ skumburegama@yahoo.com (corresponding author)
}

\begin{abstract}
The present study was carried out in the recently established Warathenna-Hakkinda EPA in the Kandy District, Sri Lanka to investigate the avifaunal diversity and conservation threats. Sampling was conducted in two main habitat types: river islands and riverine forests. Both point count (10 m radius) and line transect ( $200 \mathrm{~m}$ long) methods were utilized and maximum of 30 minutes was used to sample the birds in each point. Food habit, niche type, endemism, abundance, and diversity indices (Shannon \& Margalef) were calculated to compare the two habitats in this area. A total of 74 bird species belonging to 61 genera and 35 families were encountered from the study site. Among these, seven species are endemic: Sri Lanka Grey Hornbill, Sri Lanka Green Pigeon, Sri Lanka Wood Pigeon, Sri Lanka Hanging Parrot, Crimson-fronted Barbet, Yellow-fronted Barbet, and Sri Lanka Hill Mynah; while six were migratory: Green Sandpiper, Common Sandpiper, Indian Pitta, Asian Brown Flycatcher, Yellow Wagtail, and Grey Wagtail and the rest were non-endemic natives. Most of them were canopy and sub canopy dwellers associated with riverine forests and islands. According to the National Red List of Sri Lanka, three species, the Sri Lanka Wood Pigeon, Sri Lanka Hill Mynah, and Alpine Swift, are listed as threatened. Ardeidae, Alcedinidae, Columbidae, Accipitridae, and Apodidae were the most species rich families in this habitat. Out of the sampled species, $31 \%$ and $25 \%$ of the birds were carnivores and insectivores, respectively, while $5 \%$ were nectarivores. According to the avifauna, the riverine forests are more diverse (Shannon index $H^{\prime}=2.55$; Margalef's index $M=10.92$ ) than the river islands $\left(H^{\prime}=2.29 ; M=5.07\right)$ in this landscape. The variety of habitats along the Mahaweli River at Warathenna appears to aid in sustaining a rich bird community and this Environmental Protection Area will help create a safe haven for the birds.
\end{abstract}

Keywords: Birds, conservation threats, endemic species, riverine forests, Kandy District, river islands, Warathenna-Hakkinda.

Citation: Thilakarathne, D., T. Lakkana, G. Hirimuthugoda, C. Wijesundara \& S. Kumburegama (2021).Diversity and distribution of avifauna at Warathenna-Hakkinda Environmental Protection Area in Kandy, Sri Lanka. Journal of Threatened Taxa 13(12): 19689-19701. https://doi.org/10.11609/jott.7447.13.12.19689-19701

Copyright: (c) Thilakarathne et al. 2021. Creative Commons Attribution 4.0 International License. JoTT allows unrestricted use, reproduction, and distribution of this article in any medium by providing adequate credit to the author(s) and the source of publication.

Funding: Biodiversity Secretariat, Ministry of Mahaweli Development and Environment, Sri Lanka.

Competing interests: The authors declare no competing interests.

Author details: DinelKa Thilakarathne (B.Sc. and M.Phil.) is a Lecturer (Probationary) in the Department of Zoology, Faculty of Science, University of Ruhuna. Animal ecology and wildlife conservation are her main research interests and she is currently conducting biodiversity and animal ecology related research in Sri Lanka. TITHIRA LAKKANA is a Research Assistant attached to the China-Sri Lanka Joint Center for Research and Education (CSL-CER), University of Ruhuna. He completed his M.Phil. (Forestry) in 2020 and is currently involved in the mangrove conservation project in Sri Lanka. His research interests are in plant regeneration and forest ecology. GAYAN HIRIMUTHUGODA is a Technical Officer at the Department of Zoology, University of Peradeniya. In reltation to his interests in biodiversity, he is currntly engaged in research work related to fish breeding, embryology and rearing, especially of endemic, Endangered species. CHAMINDA WIJESUNDARA is a Senior Lecturer in the Department of Zoology, University of Peradeniya, Sri Lanka. He is currently engaged in research on waterbirds and songbirds. SHALIKA KUMBUREGAMA is a developmental biologist with a Ph.D. in Zoology from University of Hawaii at Manoa, USA. She is a Senior Lecturer in the Department of Zoology, University of Peradeniya, Sri Lanka and is involved in studies in embryology and developmental biology, and biodiversity and conservation.

Authors contributions: DK-research design, data collection, species identification, data analysis and manuscript writing. TL-research design, data collection, species identification, data analysis and manuscript reviewing. GH-research design, data collection, species identification and manuscript writing and reviewing. $\mathrm{CW}-$ species Identification, data analysis, manuscript reviewing. SK-research planning and designing, data collection, manuscript writing and reviewing. Principal Investigator of the research.

Acknowledgements: This work was supported by the Small Grant Research Program (2016) of the Biodiversity Secretariat, Ministry of Mahaweli Development and Environment, Sri Lanka. 


\section{INTRODUCTION}

Sri Lanka and the Western Ghats of India is considered a global biodiversity hotspot due to the high diversity and species endemicity (Bossuyt et al. 2004). Sri Lanka, which is a tropical island located in the Indian Ocean between longitudes $79.65 \mathrm{E}$ and $81.88 \mathrm{E}$ and latitudes $5.90 \mathrm{~N}$ and $9.86 \mathrm{~N}$ experiences a year round tropical climate with average temperatures of $27-28{ }^{\circ} \mathrm{C}$ and a relatively constant day length (Wijesundara \& de Silva 2005). The precipitation in the island is largely governed by the south-west and north-east monsoons (Ashton et al. 1997; Gunatilleke et al. 2008). Sri Lanka is renowned for the diversity and endemicity of its vertebrate fauna, including its diverse avifauna (de Silva Wijeyeratne 2014; Weerakoon 2015).

Sri Lanka is home to 240 breeding resident species, 216 purely migrant species, and 72 vagrant species of birds. Of the total 453 species, 27 are endemic, and six are proposed as endemics (Gunawardena \& Weerakoon 2012), while 43 species, including five winter migrants are considered endemic to the southern Asian region (Kotagama \& Wijeyasinghe 1998; Kotagama 2000; Santiapillai \& Wijesundara 2002; Rasmussen \& Anderson 2005; Wijesundara \& de Silva 2005; Chandrasiri et al. 2018). According to the National Red List (MOE 2012), 46 of the breeding species on the island are nationally threatened, including 10 Critically Endangered, 15 Endangered, and 21 Vulnerable bird species. The country is divided into six avifaunal zones and two sub zones by considering the distribution patterns of the resident birds. The six major avifaunal zones are Highlands Avifaunal Zone, Wet Forest Avifaunal Zone, Uva Avifaunal Zone, Marine Avifaunal Zone, Palk Bay Coastal Avifaunal Zone, and Rakwana Hill Avifaunal Zone. The two sub zones are Wet Lowland Avifaunal Sub-Zone, and Wet Mid-Hill Avifaunal Sub-Zone (Kotagama 1993; MoMD\&E 2019). This study was conducted to investigate the avifaunal diversity in the Warathenna-Hakkinda Environmental Protection Area (EPA) in the Kandy district located in the Wet Forest Avian Zone (Harrison \& Worfolk 1999; MoMD\&E 2019).

Warathenna-Hakkinda EPA was declared in 2017 after the findings of rare, endemic flora and fauna from this area. This EPA has an extent of $0.61 \mathrm{~km}^{2}$ extending from the Halloluwa suspension bridge in the north to the Gatambe Bridge in the south, and bordered by the Peradeniya-Halloluwa-Katugastota Road on one side and the "Srimath Kuda Rathwaththe Mawatha", "Deveni Rajasinghe Mawatha", and the Colombo-Kandy Road on the other side of the river (Sri Lanka 2017:
4/81.VI). This EPA encompasses an approximately $2 \mathrm{~km}$ stretch of the Mahaweli River, all its river islands in this stretch referred to as the Hakkinda river islands, as well as the surrounding riverine forests and Kandyan home gardens. Only a handful of studies thus far have looked at the biodiversity in this area including the diversity of dragonflies and fish (Samarawickrama et al. 2012; Kumara \& Samarawickrama 2017; Thilakarathne et al. 2019). The present study aimed to evaluate the species composition of the bird community in this EPA

\section{MATERIALS AND METHODS}

\section{Description of the study site}

Mahaweli river is the longest river in Sri Lanka. It originates from the central highlands and flows through the wet, intermediate and dry zones of the country (Fernando 1990). The study was conducted along a 2 $\mathrm{km}$ stretch of the Mahaweli River in the Kandy District starting from the Gatambe main bridge (7.45N, $80.10 \mathrm{E})$ to the Dodangwela Suspension Bridge (7.46N, 80.10E), from June 2016 to May 2017. This area falls within the Warathenna-Hakkinda Environmental Protection Area (EPA). The banks of the Mahaweli River along this stretch of the study site consists of riverine forests patches and river islands that were distinguished according to the geomorphological features and the vegetation characteristics.

Vegetation of the riverine forests and river islands consists of both native endemic and non-endemic plant species as well as higher number of exotic species due to the high influence of anthropogenic activities. In the riverine forests, plant species such as, Terminalia arjuna, Neolitsea cassia, Mesua thwaitesii, Dillenia indica, Barringtonia racemosa, Horsfieldia iryaghedhi, Glochidion zeylanicum, Ficus tsjahela, Ficus benghalensis, Ficus racemosa, Symplocos cochinchinensis, Spathodea campanulata, Alstonia macrophylla, Michelia champaca, Caryota urens, and Bambusa vulgaris were frequently encountered. In the approximately 20 river islands in the EPA (the largest of which has an extent of about 3.2 ha), plant species such as Leea indica, Schefflera stellata, Ficus hispida, Ficus tinctoria, Ficus exasperata, Pandanus kaida, Syzygium jambos, Mimosa pigra, Clusia rosea, T. arjuna, D. indica, M. thwaitesii, N. cassia, Terminalia catappa, and Macaranga peltata were recorded. Members of the genera Lagenandra (L. praetermissa) and Cryptocoryne (C. undulata, C. beckettii, C. walkeri, and $C$. parva) were seen in the shallow waters around the river islands. Furthermore, the exotic free floating 




Figure 1. Map of Warathenna-Hakkinda EPA starting from the Gatambe main bridge $(7.45 \mathrm{~N}, 80.10 \mathrm{E})$ to the Dodangwela Suspension Bridge (7.46N, 80.10E).

Eichhornia crassipes was seen in areas with slow moving water.

The sampling of avifauna was carried out in 17 river islands and 24 riverine forest patches. Sampling locations were marked using a portable global positioning system (GPS) (Magellan eXplorist 110) and sampling points were separated by a minimum distance of $150 \mathrm{~m}$ (Figure 1).

\section{Survey of avian fauna along the declared EPA}

Field sampling was carried out twice a week during the study period. Systematic random sampling method was used to record the birds from this EPA, and sampling was conducted along two main roads on either side of the Mahaweli River, the Peradeniya-HalloluwaKatugastota Road $(2.5 \mathrm{~km}$ ) on the west and the "Srimath Kuda Rathwaththe Mawatha" and "Deveni Rajasinghe Mawatha" (3 km) on the east. Eighteen $200 \mathrm{~m}$ line 
transacts were sampled along the two main roads within the EPA. Two $10 \mathrm{~m}$ radius points along each $200 \mathrm{~m}$ line transect were established to record the birds along the riverine forest. A point count method ( $5 \mathrm{~m}$ radius) was carried out in the river islands and along river banks to record bird species. A maximum of 30 minutes was spent in each site during both point count and line transect methods. Birds were observed using direct observations with the aid of $10 \times 42$ binoculars (Nikon, Monarch) and identified using available field guides (e.g., Harrison, 2011; Warakagoda et al. 2012) as well as through vocalizations. Opportunistic and incidental observations were also recorded during the survey. With the aid of previously published records (Henry 1971; Ali \& Ripley 2001), the recorded birds were categorized according to their dietary habits. The conservation status of identified bird species was classified according to the National Red List 2012 of Sri Lanka (MOE 2012). The counting was conducted at 0630-0930 $\mathrm{h}$ and $1530-1830 \mathrm{~h}$ when most of the birds are active. The bird species, their abundance, and forest strata where they were observed were recorded in the field.

\section{Data analysis}

Data on avifauna were analyzed for riverine forests and the river islands. The species richness, family richness, endemism, migratory percentages, relative abundance, and the diversity were calculated using $R$ version 3.6.1 ( $R$ Core Team 2014). Pooled t-test was carried out to determine the significance of the two habitats under $95 \%$ confidant intervals using $\mathrm{R}$ version 3.6.1 ( $R$ Core Team 2014). The difference among different micro-habitats/strata was evaluated using one-way ANOVA using tukey comparison under 95\% confidant limits in R studio version 3.6.1 (R Core Team 2014). The relative abundance was calculated as follows.

Relative abundance $\left(p_{i}\right)=\frac{\text { Number of individuals in a particular species }}{\text { Total Number of sampled individuals in all species }}$

The rarefaction curve, endemism, family representation and national conservation status were plotted using SigmaPlot-10. Chao 2 and second order Jacknife 2 richness estimators were calculated in EstimateS software (Version 9.1.0) (Chao et al. 2005) to estimate the predicted species richness in the EPA. Species diversity was measured using Shannon diversity index. The proportional abundance of species was used to determine the Shannon diversity index $\left(\mathrm{H}^{\prime}\right)$ and the species richness was used to determine Margalef's diversity index using $R$ version 3.6.1 (R Core Team 2014) for comparison of avifaunal diversity between riverine forests and river islands. Jaccard similarity index was calculated using $\mathrm{R}$ version 3.6.1 ( $\mathrm{R}$ Core Team 2014) to estimate the degree of similarity between the two habitat types.

$$
\begin{gathered}
\text { Shannon - Weiner index }\left(H^{\prime}\right)=-\sum p_{i} \ln p_{i} \\
\text { Margaleaf index }(\mathrm{M})=\frac{S-1}{\ln N}
\end{gathered}
$$

Where, $\mathrm{p}_{\mathrm{i}}=$ Relative abundance of a species, $\mathrm{S}=$ Number of species (species richness), and $\mathrm{N}=$ Total number of individuals

$$
\text { Jaccard Index }\left(C_{j}\right)=\frac{j}{(a+b-j)}
$$

Where, $j=$ Number of species found in both sites, $a=$ Number of species in site $A$, and $b=$ Number of species in site B

\section{RESULTS}

A total of 666 individuals belonging to 74 bird species, 61 genera and 35 families were encountered during the study (Table 1, Image 1,2). The species based rarefaction curve, with lower and upper limits, did not reach the asymptote, which implied that more sampling effort was needed. According to the Chao 2 and Jackknife 2 estimates, the species richness could be 88 and 104, respectively (Figure 2A,B). Seven species: Yellow-fronted Barbet Psilopogon flavifrons, Crimson-fronted Barbet Psilopogon rubricapillus, Sri Lanka Green Pigeon Treron pompadora, Sri Lanka Wood Pigeon Columba torringtoniae, Sri Lanka Hill Mynah Gracula ptilogenys, Sri Lanka Hanging Parrot Loriculus beryllinus, and Sri Lanka Grey Hornbill Ocyceros gingalensis (Image 2A) encountered during the study are endemic, while six: Grey Wagtail Motacilla cinerea, Yellow Wagtail Motacilla flava, Asian Brown Flycatcher Muscicapa dauurica, Indian Pitta Pitta brachyura (Image 21), Common Sandpiper Actitis hypoleucos, and Green Sandpiper Tringa ochropus) are migratory (Figure 3). However, majority of the sampled birds were nonendemic native resident birds. Since this is a riverine system, $24 \%$ of the encountered species were water associated birds while $76 \%$ were terrestrial birds. The most species-rich family was Ardeidae (herons and egrets; Image $1 \mathrm{C}, \mathrm{E}, \mathrm{F})$ with seven species. Other than that, Alcedinidae (kingfishers - four species; Image 1A, $B)$, Columbidae (pigeons - five species), Accipitridae (raptors - four species), and Apodidae (swifts - four species) were the most common families represented in the EPA. Of the recorded families, $43 \%$ were represented by only a single species. Although the species richness 

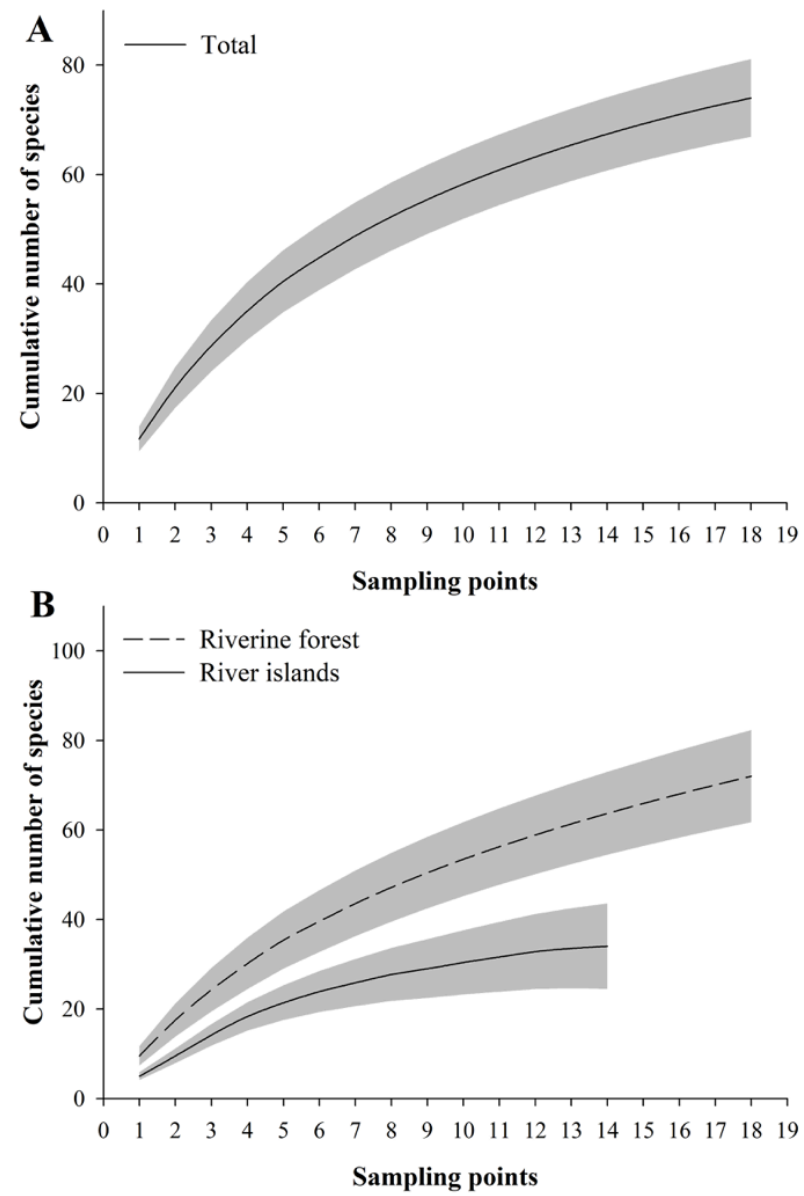

Figure 2. Species based rarefaction representation of the birds recorded from Warathenna-Hakkinda EPA from 2016 to 2017 with 95\% lower and upper boundaries: A-Total species richness | BSpecies richness between riverine forests and river islands.

was highest in Ardeidae, Columbidae, Alcedinidae, Accipitridae, and Apodiade families, the highest number of individuals in this ecosystem and hence the dominant families were Aredeidae (15\% of relative abundance), Phalacrocoracidae ( $12 \%$ of relative abundance), and Sturnidae ( $11 \%$ of relative abundance; Table 1 ).

Out of the total number of species encountered, three species are listed under the categories of Vulnerable (VU) and Endangered (EN) according to the National Red List (MOE 2012). Of the remaining species, three are listed as Near Threatened (NT) and the rest of the 63 species are listed as Least Concern (LC). Of the recorded species, Alpine Swift is in the EN category while the Sri Lanka Wood Pigeon and Sri Lanka Mynah are listed as VU (MOE 2012). However, according to the global conservation status, the Sri Lanka Wood Pigeon is considered to be VU, Sri Lanka Hill Mynah as NT while the rest of the 72 species are considered to be LC. The LC category species were the most abundant $199 \%$ of

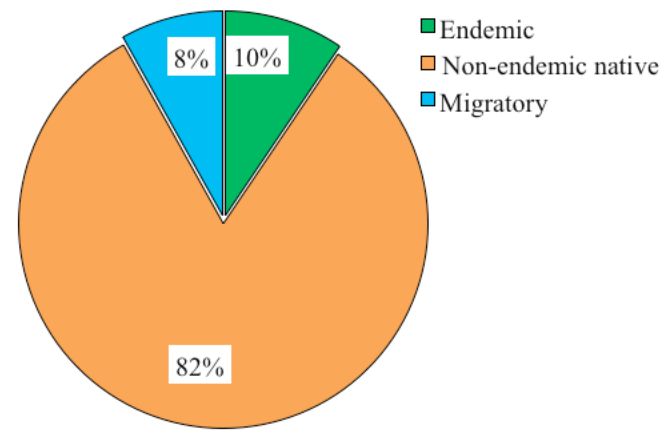

Figure 3. Endemism of the birds recorded from WarathennaHakkinda EPA

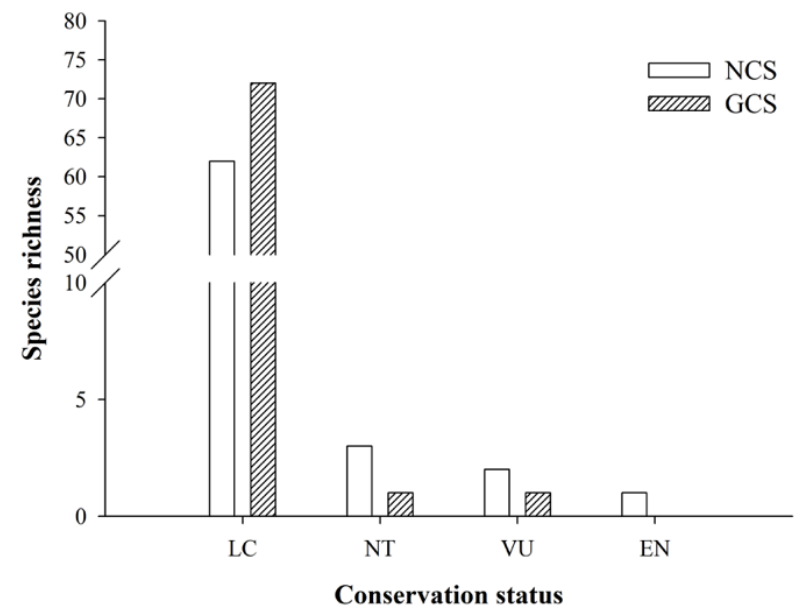

Figure 4. National conservation status (NCS) and Global conservation status (GCS) of the bird species recorded from Warathenna-Hakkinda EPA: LC-Least Concern | NT-Near Threatened | VU-Vulnerable | EN-Endangered.

relative abundance) in this ecosystem while $\mathrm{VU}$ species were the least abundant $(0.3 \%$ of relative abundance; Table 1 and Figure 4).

According to the food habits, the encountered birds in the EPA included carnivores, omnivores, insectivores, piscivores, frugivores, nectarivores, and granivores. The species richness of the birds among the food habit categories was significantly different $(F=1.45 ; p<0.05)$. Of these, most species were categorized as carnivores (31\%) and insectivores (25\%) while nectarivores (5\%) were the lowest in this riverine system. In addition, the highest numbers of individuals were recorded from carnivores and omnivores (35\% and $26 \%$ of relative abundance, respectively) while the least number of individuals were recorded from nectarivores (Figure 5).

Forests can be defined by four vertical forest strata namely, canopy, sub canopy, understory and the ground layer. Some of the bird species in the EPA were encountered from a single layer while others were 
Table 1. Recorded bird species, their conservation status, forest strata, food habit and relative abundance in riverine forests and river islands in Warathenna-Hakkinda EPA, Kandy.

\begin{tabular}{|c|c|c|c|c|c|c|c|c|c|c|}
\hline \multirow[b]{2}{*}{ Family } & \multirow[b]{2}{*}{ Scientific Name } & \multirow[b]{2}{*}{ Common Name } & \multirow{2}{*}{$\begin{array}{l}\text { NCS/ } \\
\text { GCS }\end{array}$} & \multirow[b]{2}{*}{ Stratum } & \multirow[b]{2}{*}{ Food habit } & \multirow[b]{2}{*}{ RA/\% } & \multirow[b]{2}{*}{ Rarity } & \multicolumn{3}{|c|}{ Abundance } \\
\hline & & & & & & & & RI & RF & $p$ Value \\
\hline Accipitridae & Accipiter badius & Shikra & $\mathrm{LC} / \mathrm{LC}$ & Canopy & Carnivores & 0.3 & $\mathrm{R}$ & 1 & 1 & - \\
\hline Accipitridae & Haliaeetus leucogaster & $\begin{array}{l}\text { White-bellied Sea } \\
\text { Eagle }\end{array}$ & $\mathrm{LC} / \mathrm{LC}$ & Canopy & Carnivores & 0.6 & c & 2 & 2 & - \\
\hline Accipitridae & Haliastur indus & Brahminy Kite & $\mathrm{LC} / \mathrm{LC}$ & Canopy & Carnivores & 0.9 & c & 2 & 4 & - \\
\hline Accipitridae & Spilornis cheela & Crested Serpent Eagle & $\mathrm{LC} / \mathrm{LC}$ & Canopy & Carnivores & 0.6 & c & 1 & 3 & - \\
\hline Alcedinidae & Alcedo atthis & Common Kingfisher & $\mathrm{LC} / \mathrm{LC}$ & $\begin{array}{l}\text { Sub canopy, } \\
\text { Canopy }\end{array}$ & Carnivores & 0.3 & $\mathrm{R}$ & 0 & 2 & - \\
\hline Alcedinidae & Ceyx erithaca & $\begin{array}{l}\text { Oriental Dwarf } \\
\text { Kingfisher }\end{array}$ & NT/LC & $\begin{array}{l}\text { Sub canopy, } \\
\text { Canopy }\end{array}$ & Carnivores & 0.3 & $\mathrm{R}$ & 1 & 1 & - \\
\hline Alcedinidae & Halcyon smyrnensis & $\begin{array}{l}\text { White-throated } \\
\text { Kingfisher } \\
\end{array}$ & $\mathrm{LC} / \mathrm{LC}$ & \begin{tabular}{|l} 
Sub canopy, \\
Canopy
\end{tabular} & Carnivores & 1.2 & c & 1 & 7 & - \\
\hline Alcedinidae & Pelargopsis capensis & Stork-billed Kingfisher & $\mathrm{LC} / \mathrm{LC}$ & \begin{tabular}{|l} 
Sub canopy, \\
Canopy
\end{tabular} & Carnivores & 1.95 & C & 4 & 9 & 0.54 \\
\hline Apodidae & Aerodramus unicolor & Indian Swiftlet & $\mathrm{LC} / \mathrm{LC}$ & $\begin{array}{l}\text { Canopy, Sub } \\
\text { canopy }\end{array}$ & Insectivores & 0.75 & C & 3 & 2 & - \\
\hline Apodidae & Apus melba & Alpine Swift & $\mathrm{EN} / \mathrm{LC}$ & $\begin{array}{l}\text { Canopy, Sub } \\
\text { canopy }\end{array}$ & Insectivores & 0.6 & C & 0 & 4 & - \\
\hline Apodidae & Apus nipalensis & House Swift & $\mathrm{LC} / \mathrm{LC}$ & $\begin{array}{l}\text { Canopy, Sub } \\
\text { canopy }\end{array}$ & Insectivores & 1.5 & C & 0 & 10 & - \\
\hline Apodidae & Cypsiurus balasiensis & Asian Palm-Swift & $\mathrm{LC} / \mathrm{LC}$ & $\begin{array}{l}\text { Canopy, Sub } \\
\text { canopy }\end{array}$ & Insectivores & 0.45 & C & 0 & 3 & - \\
\hline Ardeidae & Ardea alba & Great Egret & $\mathrm{LC} / \mathrm{LC}$ & $\begin{array}{l}\text { Sub canopy, } \\
\text { Ground }\end{array}$ & Carnivores & 0.15 & $\mathrm{R}$ & 0 & 1 & - \\
\hline Ardeidae & Ardea intermedia & Intermediate Egret & $\mathrm{LC} / \mathrm{LC}$ & $\begin{array}{l}\text { Sub canopy, } \\
\text { Ground }\end{array}$ & Carnivores & 5.26 & VC & 5 & 30 & 0.27 \\
\hline Ardeidae & Ardeola grayii & Indian Pond Heron & $\mathrm{LC} / \mathrm{LC}$ & $\begin{array}{l}\text { Sub canopy, } \\
\text { Ground }\end{array}$ & Carnivores & 0.6 & c & 0 & 4 & - \\
\hline Ardeidae & Bubulcus ibis & Cattle Egret & $\mathrm{LC} / \mathrm{LC}$ & $\begin{array}{l}\text { Sub canopy, } \\
\text { Ground }\end{array}$ & Carnivores & 3 & C & 10 & 10 & 0.61 \\
\hline Ardeidae & Egretta garzetta & Little Egret & $\mathrm{LC} / \mathrm{LC}$ & $\begin{array}{l}\text { Sub canopy, } \\
\text { Ground }\end{array}$ & Carnivores & 5.41 & VC & 11 & 25 & 0.34 \\
\hline Ardeidae & $\begin{array}{l}\text { Ixobrychus } \\
\text { cinnamomeus }\end{array}$ & Cinnamon Bittern & NT/LC & $\begin{array}{l}\text { Sub canopy, } \\
\text { Ground }\end{array}$ & Carnivores & 0.15 & $\mathrm{R}$ & 0 & 1 & - \\
\hline Ardeidae & Nycticorax nycticorax & $\begin{array}{l}\text { Black-crowned Night } \\
\text { Heron }\end{array}$ & NT/LC & Ground & Carnivores & 0.3 & $\mathrm{R}$ & 1 & 1 & - \\
\hline Bucerotidae & Ocyceros gingalensis ${ }^{E}$ & Sri Lanka Grey Hornbill & $\mathrm{LC} / \mathrm{LC}$ & $\begin{array}{l}\text { Sub canopy, } \\
\text { Canopy }\end{array}$ & Frugivores & 0.3 & $\mathrm{R}$ & 1 & 1 & - \\
\hline Campephagidae & \begin{tabular}{|l}
$\begin{array}{l}\text { Pericrocotus } \\
\text { cinnamomeus }\end{array}$ \\
\end{tabular} & Small Minivet & $\mathrm{LC} / \mathrm{LC}$ & \begin{tabular}{|l} 
Sub canopy, \\
Understory \\
\end{tabular} & Insectivores & 0.6 & c & 0 & 4 & - \\
\hline Campephagidae & Pericrocotus speciosus & Scarlet Minivet & $\mathrm{LC} / \mathrm{LC}$ & \begin{tabular}{|l} 
Sub canopy, \\
Understory
\end{tabular} & Insectivores & 1.65 & C & 5 & 6 & 0.52 \\
\hline Charadriidae & Vanellus indicus & Red-wattled Lapwing & $\mathrm{LC} / \mathrm{LC}$ & Ground & Omnivores & 0.6 & C & 0 & 4 & - \\
\hline Chloropseidae & Chloropsis aurifrons & $\begin{array}{l}\text { Golden-fronted } \\
\text { Leafbird }\end{array}$ & $\mathrm{LC} / \mathrm{LC}$ & \begin{tabular}{|l} 
Sub canopy, \\
Understory
\end{tabular} & Frugivores & 0.6 & C & 2 & 2 & - \\
\hline Chloropseidae & Chloropsis jerdoni & Jerdon'sLeafbird & $\mathrm{LC} / \mathrm{LC}$ & $\begin{array}{l}\text { Sub canopy, } \\
\text { Understory } \\
\end{array}$ & Frugivores & 0.75 & C & 2 & 3 & - \\
\hline Cisticolidae & Prinia inornata & Plain Prinia & $\mathrm{LC} / \mathrm{LC}$ & \begin{tabular}{|l} 
Sub canopy, \\
Understory \\
\end{tabular} & Insectivores & 0.3 & $\mathrm{R}$ & 0 & 2 & - \\
\hline Cisticolidae & Prinia socialis & Ashy Prinia & $\mathrm{LC} / \mathrm{LC}$ & $\begin{array}{l}\text { Sub canopy, } \\
\text { Understory }\end{array}$ & Insectivores & 0.45 & C & 0 & 3 & - \\
\hline Columbidae & Chalcophaps indica & Emerald Dove & $\mathrm{LC} / \mathrm{LC}$ & $\begin{array}{l}\text { Sub canopy, } \\
\text { Understory }\end{array}$ & Frugivores & 0.3 & $\mathrm{R}$ & 0 & 2 & - \\
\hline Columbidae & Columba livia & Feral Pigeon & $-/ L C$ & $\begin{array}{l}\text { Sub canopy, } \\
\text { Understory }\end{array}$ & Granivores & 1.5 & C & 0 & 10 & - \\
\hline Columbidae & Columba torringtoniae ${ }^{E}$ & Sri Lanka Wood Pigeon & VU/VU & $\begin{array}{l}\text { Sub canopy, } \\
\text { Understory }\end{array}$ & Frugivores & 0.3 & $\mathrm{R}$ & 0 & 2 & - \\
\hline Columbidae & Streptopelia chinensis & Spotted Dove & $\mathrm{LC} / \mathrm{LC}$ & $\begin{array}{l}\text { Sub canopy, } \\
\text { Understory }\end{array}$ & Granivores & 1.05 & C & 0 & 7 & - \\
\hline Columbidae & Treron pompadora ${ }^{E}$ & Sri Lanka Green Pigeon & $\mathrm{LC} / \mathrm{LC}$ & $\begin{array}{l}\text { Sub canopy, } \\
\text { Understory }\end{array}$ & Frugivores & 0.3 & $\mathrm{R}$ & 0 & 2 & - \\
\hline
\end{tabular}




\begin{tabular}{|c|c|c|c|c|c|c|c|c|c|c|}
\hline \multirow[b]{2}{*}{ Family } & \multirow[b]{2}{*}{ Scientific Name } & \multirow[b]{2}{*}{ Common Name } & \multirow{2}{*}{$\begin{array}{l}\text { NCS/ } \\
\text { GCS }\end{array}$} & \multirow[b]{2}{*}{ Stratum } & \multirow[b]{2}{*}{ Food habit } & \multirow[b]{2}{*}{ RA/\% } & \multirow[b]{2}{*}{ Rarity } & \multicolumn{3}{|c|}{ Abundance } \\
\hline & & & & & & & & $\mathbf{R I}$ & RF & $p$ Value \\
\hline Corvidae & Corvus splendens & House Crow & LC/LC & $\begin{array}{l}\text { Sub canopy, } \\
\text { Understory }\end{array}$ & Omnivores & 7.81 & VC & 5 & 47 & 0.09 \\
\hline Cuculidae & Centropus sinensis & Greater Coucal & LC/LC & \begin{tabular}{|l} 
Understory, \\
Ground \\
\end{tabular} & Carnivores & 0.45 & C & 2 & 1 & - \\
\hline Cuculidae & Eudynamys scolopaceus & Asian Koel & $\mathrm{LC} / \mathrm{LC}$ & $\begin{array}{l}\text { Sub canopy, } \\
\text { Understory }\end{array}$ & Frugivores & 1.05 & c & 1 & 6 & - \\
\hline Dicaeidae & \begin{tabular}{|l} 
Dicaeum \\
erythrorhynchos
\end{tabular} & \begin{tabular}{|l|} 
Pale-billed \\
Flowerpecker \\
\end{tabular} & $\mathrm{LC} / \mathrm{LC}$ & \begin{tabular}{|l|} 
Sub canopy, \\
Understory \\
\end{tabular} & Nectarivores & 2.25 & c & 2 & 13 & - \\
\hline Dicruridae & Dicrurus caerulescens & White-bellied Drongo & $\mathrm{LC} / \mathrm{LC}$ & \begin{tabular}{|l|} 
Sub canopy, \\
Understory \\
\end{tabular} & Insectivores & 0.9 & C & 0 & 6 & - \\
\hline Estrildidae & Lonchura malacca & Black-headed Munia & LC/LC & \begin{tabular}{|l} 
Sub canopy, \\
Understory
\end{tabular} & Granivores & 0.75 & c & 0 & 5 & - \\
\hline Estrildidae & Lonchura punctulata & Scaly-breasted Munia & LC/LC & $\begin{array}{l}\text { Sub canopy, } \\
\text { Understory }\end{array}$ & Granivores & 2.4 & C & 0 & 16 & - \\
\hline Estrildidae & Lonchura striata & White-rumpedMunia & $\mathrm{LC} / \mathrm{LC}$ & $\begin{array}{l}\text { Sub canopy, } \\
\text { Understory }\end{array}$ & Granivores & 2.1 & c & 0 & 14 & - \\
\hline Hirundinidae & Cecropis hyperythra & Sri Lanka Swallow & $\mathrm{LC} / \mathrm{LC}$ & $\begin{array}{l}\text { Canopy, Sub } \\
\text { canopy }\end{array}$ & Insectivores & 0.45 & c & 0 & 3 & - \\
\hline Motacillidae & Motacilla cinerea ${ }^{M}$ & Grey Wagtail & $-/ L C$ & Ground & Carnivores & 0.3 & $\mathrm{R}$ & 0 & 2 & - \\
\hline Motacillidae & Motacilla flava ${ }^{M}$ & Yellow Wagtail & $-/ L C$ & Ground & Carnivores & 0.15 & R & 0 & 1 & - \\
\hline Muscicapidae & Copsychus saularis & Oriental Magpie-Robin & $\mathrm{LC} / \mathrm{LC}$ & $\begin{array}{l}\text { Sub canopy, } \\
\text { Understory }\end{array}$ & Insectivores & 0.9 & c & 0 & 6 & - \\
\hline Muscicapidae & Cyornis tickelliae & $\begin{array}{l}\text { Tickell's Blue } \\
\text { Flycatcher }\end{array}$ & $\mathrm{LC} / \mathrm{LC}$ & $\begin{array}{l}\text { Sub canopy, } \\
\text { Ground }\end{array}$ & Insectivores & 0.15 & $\mathrm{R}$ & 0 & 1 & - \\
\hline Muscicapidae & Muscicapa dauurica ${ }^{M}$ & Asian Brown Flycatcher & $-/ L C$ & Sub canopy & Insectivores & 0.15 & R & 0 & 1 & - \\
\hline Nectariniidae & Cinnyris asiaticus & Purple Sunbird & $\mathrm{LC} / \mathrm{LC}$ & $\begin{array}{l}\text { Sub canopy, } \\
\text { Understory }\end{array}$ & Nectarivores & 1.5 & c & 4 & 6 & 0.48 \\
\hline Nectariniidae & Cinnyris lotenius & Loten's Sunbird & $\mathrm{LC} / \mathrm{LC}$ & $\begin{array}{l}\text { Sub canopy, } \\
\text { Understory }\end{array}$ & Nectarivores & 0.3 & $\mathrm{R}$ & 0 & 2 & - \\
\hline Nectariniidae & Leptocoma zeylonica & $\begin{array}{l}\text { Purple-rumped } \\
\text { Sunbird }\end{array}$ & LC/LC & \begin{tabular}{|l|} 
Sub canopy, \\
Understory
\end{tabular} & Nectarivores & 1.95 & c & 3 & 10 & 0.42 \\
\hline Oriolidae & Oriolus xanthornus & Black-hooded Oriole & $\mathrm{LC} / \mathrm{LC}$ & $\begin{array}{l}\text { Sub canopy, } \\
\text { Understory }\end{array}$ & Frugivores & 1.2 & c & 1 & 7 & - \\
\hline Paridae & Parus major & Great Tit & LC/LC & $\begin{array}{l}\text { Sub canopy, } \\
\text { Understory }\end{array}$ & Omnivores & 0.75 & c & 0 & 5 & - \\
\hline Passeridae & Passer domesticus & House Sparrow & LC/LC & $\begin{array}{l}\text { Sub canopy, } \\
\text { Understory }\end{array}$ & Granivores & 0.6 & c & 0 & 4 & - \\
\hline Phalacrocoracidae & Microcarbo niger & Little Cormorant & $\mathrm{LC} / \mathrm{LC}$ & $\begin{array}{l}\text { Sub canopy, } \\
\text { Ground }\end{array}$ & Carnivores & 11.11 & VC & 37 & 37 & 0.51 \\
\hline Phalacrocoracidae & $\begin{array}{l}\text { Phalacrocorax } \\
\text { fuscicollis }\end{array}$ & Indian Cormorant & $\mathrm{LC} / \mathrm{LC}$ & $\begin{array}{l}\text { Sub canopy, } \\
\text { Ground }\end{array}$ & Carnivores & 0.45 & c & 0 & 3 & - \\
\hline Picidae & $\begin{array}{l}\text { Chrysocolaptes } \\
\text { stricklandi }\end{array}$ & $\begin{array}{l}\text { Crimson-backed } \\
\text { Goldenback }\end{array}$ & $-/ L C$ & $\begin{array}{l}\text { Sub canopy, } \\
\text { Understory }\end{array}$ & Insectivores & 0.15 & $\mathrm{R}$ & 0 & 1 & - \\
\hline Picidae & Dinopium benghalense & Lesser Goldenback & $\mathrm{LC} / \mathrm{LC}$ & $\begin{array}{l}\text { Sub canopy, } \\
\text { Understory }\end{array}$ & Insectivores & 0.15 & $\mathrm{R}$ & 0 & 1 & - \\
\hline Pittidae & Pitta brachyura ${ }^{M}$ & Indian Pitta & $\mathrm{LC} / \mathrm{LC}$ & Understory & Insectivores & 0.15 & R & 0 & 1 & - \\
\hline Psittacidae & Loriculus beryllinus ${ }^{E}$ & $\begin{array}{l}\text { Sri Lanka Hanging } \\
\text { Parrot }\end{array}$ & $\mathrm{LC} / \mathrm{LC}$ & $\begin{array}{l}\text { Sub canopy, } \\
\text { Understory }\end{array}$ & Frugivores & 0.15 & $\mathrm{R}$ & 0 & 1 & - \\
\hline Psittacidae & Psitta culakrameri & Rose-ringed Parakeet & $\mathrm{LC} / \mathrm{LC}$ & $\begin{array}{l}\text { Sub canopy, } \\
\text { Understory }\end{array}$ & Frugivores & 1.2 & c & 5 & 3 & 0.71 \\
\hline Pycnonotidae & Iole indica & Yellow-browed Bulbul & $\mathrm{LC} / \mathrm{LC}$ & $\begin{array}{l}\text { Sub canopy, } \\
\text { Understory }\end{array}$ & Omnivores & 1.2 & C & 0 & 8 & - \\
\hline Pycnonotidae & Pycnonotus cafer & Red-vented bulbul & $\mathrm{LC} / \mathrm{LC}$ & $\begin{array}{l}\text { Sub canopy, } \\
\text { Understory } \\
\end{array}$ & Omnivores & 3.15 & c & 7 & 14 & 0.19 \\
\hline Rallidae & $\begin{array}{l}\text { Amaurornis } \\
\text { phoenicurus }\end{array}$ & $\begin{array}{l}\text { White-breasted } \\
\text { Waterhen }\end{array}$ & $\mathrm{LC} / \mathrm{LC}$ & Ground & Carnivores & 0.3 & $\mathrm{R}$ & 0 & 2 & - \\
\hline Ramphastidae & Psilopogon flavifrons ${ }^{E}$ & Yellow-fronted Barbet & $\mathrm{LC} / \mathrm{LC}$ & $\begin{array}{l}\text { Canopy, } \\
\text { Understory }\end{array}$ & Frugivores & 1.2 & c & 2 & 6 & - \\
\hline Ramphastidae & \begin{tabular}{|l}
$\begin{array}{l}\text { Psilopogon } \\
\text { rubricapillus }\end{array}$ \\
\end{tabular} & $\begin{array}{l}\text { Crimson-fronted } \\
\text { Barbet }\end{array}$ & LC /LC & $\begin{array}{l}\text { Canopy, } \\
\text { Understory }\end{array}$ & Frugivores & 0.6 & c & 0 & 4 & - \\
\hline Ramphastidae & Psilopogon zeylanicus & Brown-headed Barbet & $\mathrm{LC} / \mathrm{LC}$ & \begin{tabular}{|l} 
Canopy, \\
Understory
\end{tabular} & Frugivores & 1.35 & c & 2 & 7 & - \\
\hline
\end{tabular}




\begin{tabular}{|c|c|c|c|c|c|c|c|c|c|c|}
\hline \multirow[b]{2}{*}{ Family } & \multirow[b]{2}{*}{ Scientific Name } & \multirow[b]{2}{*}{ Common Name } & \multirow{2}{*}{$\begin{array}{l}\text { NCS/ } \\
\text { GCS }\end{array}$} & \multirow[b]{2}{*}{ Stratum } & \multirow[b]{2}{*}{ Food habit } & \multirow[b]{2}{*}{ RA/\% } & \multirow[b]{2}{*}{ Rarity } & \multicolumn{3}{|c|}{ Abundance } \\
\hline & & & & & & & & $\mathbf{R I}$ & RF & $p$ Value \\
\hline Scolopacidae & Actitis hypoleucos ${ }^{M}$ & Common Sandpiper & LC/LC & Ground & Carnivores & 1.35 & C & 2 & 7 & - \\
\hline Scolopacidae & Tringa ochropus ${ }^{M}$ & Green Sandpiper & $-/ L C$ & Ground & Carnivores & 0.3 & $\mathrm{R}$ & 2 & 0 & - \\
\hline Sittidae & Sitta frontalis & $\begin{array}{l}\text { Velvet-fronted } \\
\text { Nuthatch }\end{array}$ & $\mathrm{LC} / \mathrm{LC}$ & Sub canopy & Insectivores & 0.15 & $\mathrm{R}$ & 1 & 0 & - \\
\hline Sturnidae & Acridotheres tristis & Common Mynah & $\mathrm{LC} / \mathrm{LC}$ & $\begin{array}{l}\text { Sub canopy, } \\
\text { Understory }\end{array}$ & Omnivores & 5.71 & VC & 0 & 38 & - \\
\hline Sturnidae & Gracula ptilogenys ${ }^{E}$ & Sri Lanka Hill Mynah & VU/NT & $\begin{array}{l}\text { Canopy, Sub } \\
\text { canopy }\end{array}$ & Omnivores & 0.45 & C & 2 & 1 & - \\
\hline Sturnidae & Gracula religiosa & Lesser Hill Mynah & $\mathrm{LC} / \mathrm{LC}$ & $\begin{array}{l}\text { Canopy, Sub } \\
\text { canopy }\end{array}$ & Omnivores & 5.26 & VC & 24 & 11 & 0.21 \\
\hline Sylviidae & Orthotomus sutorius & Common Tailorbird & $\mathrm{LC} / \mathrm{LC}$ & $\begin{array}{l}\text { Sub canopy, } \\
\text { Understory }\end{array}$ & Insectivores & 1.95 & C & 2 & 11 & - \\
\hline Timaliidae & Turdoides affinis & Yellow-billed Babbler & $\mathrm{LC} / \mathrm{LC}$ & $\begin{array}{l}\text { Sub canopy, } \\
\text { Understory }\end{array}$ & Insectivores & 3.9 & C & 0 & 26 & - \\
\hline Zosteropidae & Zosterops palpebrosus & Oriental White-Eye & $\mathrm{LC} / \mathrm{LC}$ & $\begin{array}{l}\text { Sub canopy, } \\
\text { Understory }\end{array}$ & Omnivores & 0.6 & C & 0 & 4 & - \\
\hline
\end{tabular}

M_Migratory birds | E-Endemic birds and rest of the birds were non-endemic native birds | NCS-National conservation status | GCS-Global conservation status | LC-Least Concern | NT-Near Threatened | EN-Endangered | VU-Vulnerable | RA-Relative abundance | R-Rare | C-Common | VC-Very common | RI-River islands | RF-Riverine forests.
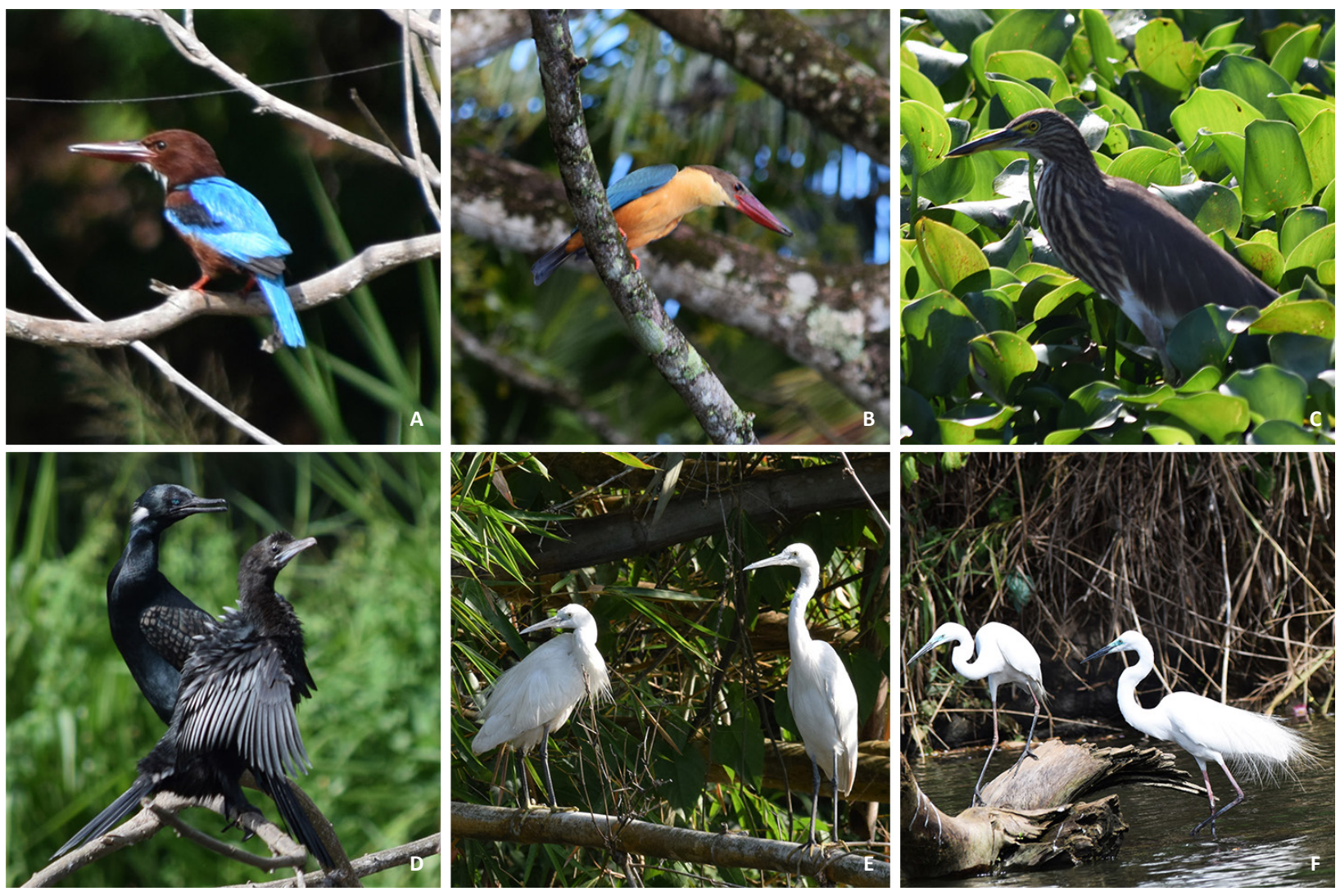

Image 1. Some of the water associated birds recorded from the Warathenna-Hakkinda EPA: A-White-throated Kingfisher | B-Stork-billed Kingfisher | C-Indian Pond Heron | D-Little Cormorant and Indian Cormorant | E-Little Egret | F-Intermediate Egret. () Tithira Lakkana. 
encountered from multiple layers such as canopy-sub canopy, canopy-understory, sub canopy-understory, sub canopy-ground and understory-ground. Most of the bird species were encountered from the canopy and sub canopy layers in the forest. According to the forest strata analysis, most of the birds were recorded from the sub canopy-understory layer ( $47 \%$ of total sampled species) while understory and understory-ground dwellers (15\% of sampled species in each strata) were the least species rich forest strata in this ecosystem. The species richness among the nine combinations of the four main strata of the forest layer was significantly different ( $\mathrm{F}=2.97$; $p$ $<0.05$ ) (Figure 6). The abundance of species was highest in sub canopy-understory (50\%) and sub canopy-ground ( $26 \%$ relative abundance) while the least abundance was recorded from the understory $(0.15 \%$ relative abundance).

During sampling of the river islands, 136 individuals belonging to 34 species in 20 families were recorded while riverine forest patches recorded 510 individuals belonging to 72 species in 34 families (Image 2B). All seven endemic species recorded during the study were observed in the riverine forests as well as six migratory species except Green Sandpiper and 60 non-endemic natives. On the other hand, three endemics (Sri Lanka Grey Hornbill, Yellow-fronted Barbet, and Sri Lanka Hill Mynah) and two migratory species (Common Sandpiper and Green Sandpiper) and 29 non-endemic natives were recorded from the river islands (Table 1). However, the species richness was not significantly different between the two habitats $(t=-2.13, p>0.05)$. Green Sandpiper and Velvet-fronted Nuthatch Sitta frontalis were the only two species not recorded from riverine forest although they were present in river islands. Swift, Munia, Wagtail, and woodpecker species were absent in the river islands in the EPA (Table 1). However, the species abundance between the two habitats were significantly different ( $\mathrm{t}=-3.89, p<0.05$ ). Yet, the relative abundance of the individual species between two habitats were not significantly different. The highly abundant species in riverine forests were the House Crow Corvus splendens, Common Mynah Acridotheres tristis, Little Cormorant Microcarbo niger (Image 1D), Intermediate Egret Ardea intermedia (Image 1F), Yellow-billed Babbler Turdoides affinis (Image 2G), and Little Egret Egretta garzetta (Image 1E) $(47,38,37,30,26$, and 25 individuals, respectively) while Little Cormorant and Southern Hill Mynah Gracula religiosa (37 and 24 individuals, respectively) were highly abundant in river islands. The least common species in riverine forest were Greater Coucal Centropus sinensis, Sri Lanka Hill Mynah, Great Egret Ardea alba, Cinnamon

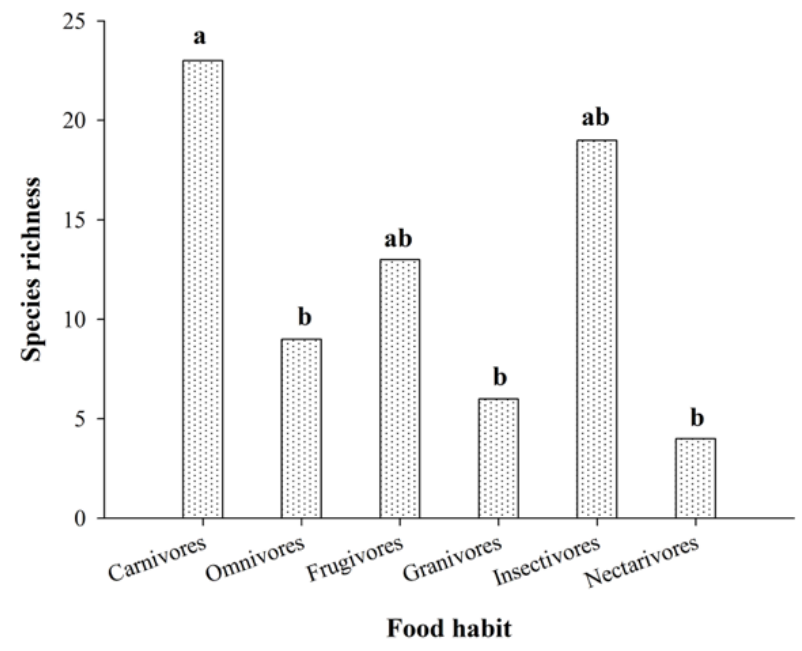

Figure 5. The food habits of sampled bird communities in Warathenna-Hakkinda EPA ( $a$ and $b$ letters denote the significance level of the data).

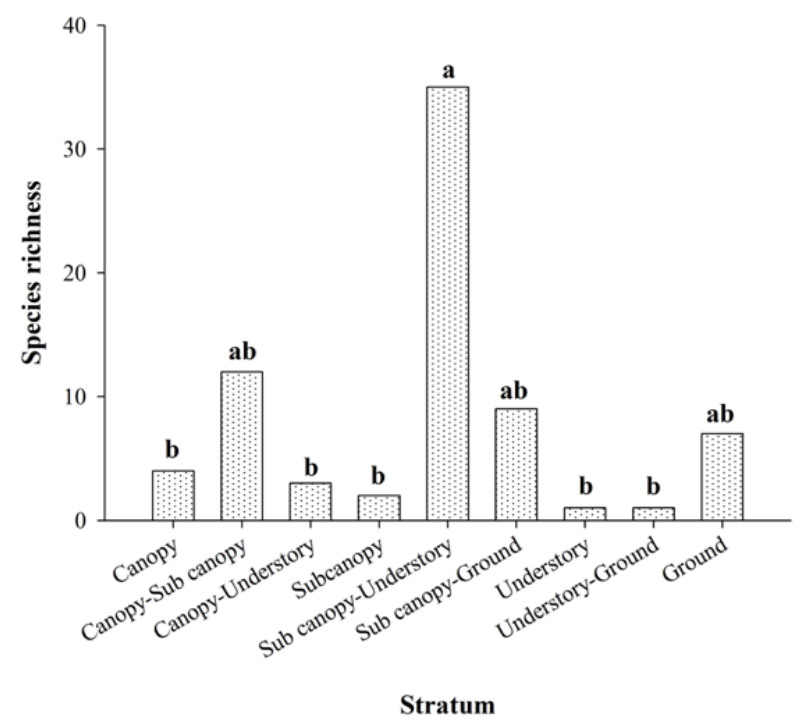

Figure 6. Distribution of encountered bird species along the forest strata in Warathenna-Hakkinda EPA; $a$ and $b$ denote the significance of the values. ( $a$ and $b$ letters denote the significance level of the data).

Bittern Ixobrychus cinnamomeus, Yellow Wagtail, Asian Brown Flycatcher, Tickell's Blue Flycatcher Cyornis tickelliae, Crimson-backed Goldenback Chrysocolaptes stricklandi, Lesser Goldenback Dinopium benghalense, Indian Pitta, and Sri Lanka Hanging Parrot while Velvetfronted Nuthatch, Crested Serpent Eagle Spilornis cheela, Asian Koel Eudynamys scolopaceus (Image 2D), Whitethroated Kingfisher Halcyon smyrnensis (Image 1A), and Black-hooded Oriole Oriolus xanthornus were the least abundant species in river islands. Shikra Accipiter badius 
(Image 2C), Oriental Dwarf Kingfisher Ceyx erithaca, Black-crowned Night Heron Nycticorax nycticorax, and Sri Lanka Grey Hornbill showed low abundance in both habitats in the EPA (Table 1).

Both Shannon-Wiener and Margalef's diversity indices were highest in riverine forests $\left(\mathrm{H}^{\prime}=2.55\right.$; $\mathrm{M}=$ 10.92) compared to the river islands $\left(H^{\prime}=2.29 ; M=\right.$ 5.07). According to the Jaccard index the two habitats showed $43 \%$ similarity. According to the food habits of the sampled species, the highest species richness and abundance was recorded from carnivores in both river island and riverine forest habitats (82 individuals 15 species and 154 individuals 22 species, respectively; Table 1), whereas the lowest species richness and abundance was recorded from nectarivores in both these habitats ( 9 individuals 3 species and 31 individuals 4 species, respectively).

\section{DISCUSSION}

Riverine forest habitats along the Mahaweli River provide shelter for diverse animal communities (Sinha et al. 2019). Due to the high heterogeneity of the habitats in riverine ecosystem, it is home to a variety of mammals, birds, reptiles, amphibians, and many invertebrate communities. However, the bird diversity in the Warathenna-Hakkinda EPA has not been investigated to date. Data on avifauna in this EPA may be useful for future conservation efforts and ecotourism.

Rarefaction graphical representations are used to determine the sampling effort. The statistical significance of this curve is revealed using 95\% upper and lower confidence limits (Colwell \& Coddington 1994). This curve for the present study is close to leveling off (i.e., not completely leveled off); hence, it indicates the requirement of more sampling effort. However, according to the published literature this graphical representation does not directly reveal the total species richness (Magurran 2004). Therefore, more commonly used and statistically powerful Chao 2 and Jackknife 2 methods are used to estimate the predicted species richness in the EPA (Colwell \& Coddington 1994; Colwell et al. 2012). Since this study was conducted during the daytime, the study possibly missed the nocturnal species and may account for the lower number of species in relation to the estimated numbers of 88 and 104 species in Chao 2 and Jackknife 2.

The 74 species of birds recorded from the EPA represents about $16 \%$ of the island's total bird species. This indicate that the species richness in this EPA is higher than those reported in many other areas around Kandy city limits (Karunaratna 1986; Wijesundara \& Santiapillai 2001; Ellepola 2014; Weerakoon 2015; Hettiarachchi \& Wijesundara 2017). However, the bird species richness in Warathenna-Hakkinda EPA is lower than what is recorded in the Gannoruwa Forest Reserve (84 species) which is located adjacent to the EPA with an extent of $2.1 \mathrm{~km}^{2}$ (Rathnayake et al. 2016). The WarathennaHakkinda EPA is a mere $0.61 \mathrm{~km}^{2}$ area with not only riverine forest and river islands but also Kandyan home gardens surrounding human habitation. Hence, this area is highly affected by anthropogenic influences compared to the Gannoruwa Forest Reserve.

This EPA harbors about $28 \%$ of the islands breeding residents (out of the 240 breeding residents in Sri Lanka) and $3 \%$ of the 213 migratory bird species in Sri Lanka. In addition, it represents $26 \%$ of endemic bird species of the island (MOE 2012; Gabadage et al. 2015), while only 11 endemic species have been recorded from the Gannoruwa forest (Rathnayake et al. 2016). The number of endemic species is a measure that can predict the amount of human influence (Linder 1995; BirdLife International 2008; Hettiarachchi \& Wijesundara 2017). Typically, undisturbed natural forests in the wet zone harbor many endemic species. For example, 16 species in the forests of the Knuckles mountain range, 12 species in the Horton Plains National Park, 23 species in the Sinharaja forest reserve, and 33 species in the Samanala Nature Reserve (Bambaradeniya et al. 2003; Karunarathna et al. 2011; Subasinghe \& Sumanapala 2014; Chandrasiri et al. 2018).

Ardeidae, Alcedinidae, some Accipitridae, and some Apodidaeare considered as water-bird families (Abie et al. 2019; Sinha et al. 2019), while Sturnidae, Estrildidae, and Columbidae families are highly associated with human habitations (Silva et al. 2017; Bellanthudawa et al. 2019). The abundance of both these two groups in the EPA hence can be correlated to its positioning along the Mahaweli River and the surrounding human settlements. Similar results have been obtained from a previous study carried out in Hantana forest, where the most abundant species was the Common Mynah, followed by Black Crow, and Yellow-billed Babbler (Wijesundara \& Santiapillai 2001). On the other hand, most of the other wet zone forest as well as the forest located within the Kandy city limits (Udawattakele Forest Reserve) has more forest dwelling individuals and species such as Swallows, Swifts, Barbets, forest Babblers, Bulbuls, and Hill Mynahs (Bambaradeniya et al. 2003; Wijesundara \& Silva 2005; Karunarathna et al. 2011; Subasinghe \& Sumanapala 2014; Rathnayake et al. 



Image 2. Some of the terrestrial endemic, non-endemic native and migratory birds recorded from the Warathenna-Hakkinda EPA: A-Sri Lanka Grey Hornbill | B-Yellow-browed Bulbul | C-Shikra | D-Asian Koel | E-House Sparrow | F-Feral Pigeon | G-Yellow-billed Babbler | HPale-billed Flowerpecker | I-Indian Pitta. @ Tithira Lakkana.

2016; Hettiarachchi \& Wijesundara 2017; Chandrasiri et al. 2018).

The forest strata the birds inhabit correlate with their food habits. Most of the frugivores (leaf birds, barbets and hill mynahs) and insectivores are observed in the canopy and sub canopy forest layers while most of the piscivores are found on the ground layer and omnivores are encountered from sub canopy, understory and ground layers during the present survey. A large number of carnivores, are recorded from the EPA because this is a water associated habitat. But our findings were contrary to the study carried out in Udawatta-kele, which is a secondary forest in Kandy (Ellepola 2014; Weerakoon 2015) and the Knuckles range (Subasinghe \& Sumanapala 2014), where they record a large number of insectivores and frugivores. A previous study carried out in the hill country of Sri Lanka documented that the insectivores (Sri Lanka Bush Warbler, Common Tailorbird), frugivores (Yellow-fronted Barbet), and nectarivores (Purple Sunbird) showed a marked 
preference for primary montane forests while carnivores preferred primary montane forests and grassland-forest interface where they can easily spot their prey species (Wijesundara \& Silva 2005).

Although there is a difference in both the diversity and distribution of birds between the two habitats studied in the EPA, a large number of common bird species (43\% of sampled birds) are recorded from both the habitats. This is possibly because there is very little distinction between these two habitats in terms of floristic characteristics. Several studies have shown that the bird distribution and diversities highly depend on the heterogeneity of the habitats (Bambaradeniya et al. 2003; Wijesundara \& Silva 2005; Karunarathna et al. 2011; Subasinghe \& Sumanapala 2014; Weerakoon 2015; Rathnayake et al. 2016; Hettiarachchi \& Wijesundara 2017; Chandrasiri et al. 2018). The higher bird diversity in the riverine forests in the EPA may be attributed to the availability of relatively more distinct habitats.

Since the Warathenna-Hakkinda EPA is surrounded by human habitation, a number of anthropogenic activities have a direct impact on the EPA. Construction and expansion of human habitation, logging, garbage, and sewage dumping can lead to the deterioration and loss of biodiversity in this EPA. Given the high diversity of flora and fauna, including birds, as indicated in this study, this area requires effective conservation planning, including proper landscape management strategies, strengthening of existing national environmental rules and policies, continuous monitoring programs, public education and awareness programs for safeguarding the biodiversity in this EPA.

\section{CONCLUSIONS}

A total of 74 bird species belonging to 61 genera and 35 families were encountered from the Warathennahakkinda EPA, which represent $16 \%$ of the island total bird species, $28 \%$ of the islands breeding residents, $3 \%$ of the migratory bird species and $26 \%$ of endemic birds of Sri Lanka. The variety of habitats provided by the riverine forests and river islands along the Mahaweli River of the EPA appears to aid in sustaining a rich bird community. However, since this EPA is located within the densely populated city of Kandy, it faces immense population pressures. Therefore, effective conservation planning including continuous monitoring programs, proper landscape managing strategies, strengthening of existing environmental policies, as well as educational and awareness programs are essential to minimize threats and safeguard the biodiversity in the EPA.

\section{REFERENCES}

Abie, K., B. Tilahun, A. Feyisa, T. Kumssa \& A. Amare (2019). Bird species diversity and distribution in case of protected area. Species 20: 90-100.

Ali, S.I. \& S.D. Ripley (2001). Handbook of the Birds of India and Pakistan together with those of Bangladesh, Nepal, Bhutan, and Sri Lanka (10 Vols.). $2^{\text {nd }}$ Edition. Oxford University Press, Delhi.

Ashton, M.S., S. Gunatilleke, N. De Zoysa, M.D. Dassanayake, N. Gunatilleke \& S. Wijesundera (1997). A Field Guide to the Common Trees and Shrubs of Sri Lanka. WHT Publication (Pvt.) Ltd for the Wildlife Heritage Trust of Sri Lanka, 95 Cotta Road, Colombo 8, Sri Lanka.

Bambaradeniya, C.N.B., M.S.J. Perera, W.P.N. Perera, L.J.M. Wickramasinghe, L.D.C.B. Kekulandala, V.A.P. Samarawickrema, R.H.S.S. Fernando \& V.A.M.P.K. Samarawickrema (2003). Composition of faunal species in the Sinharaja world Heritage site in Sri Lanka. The Sri Lanka Forester 26: 21-40.

Bellanthudawa, B.K.A., N.M.S.K. Nawalage, S. Subanky, P.A.B.G. Panagoda, H.W.G.A.S. Weerasinghe, L.K.D.N. Tharaka, H.M.A.K. Handapangoda, H.K.A.D. Silva, D.M.S.N. Dissanayake \& M.S.J. Abeywickrama (2019). Composition and Diversity Variation of Avifauna, along Different Vegetative Habitat Types in a HumanModified Area, University of Kelaniya, Sri Lanka. Hindawi International Journal of Zoology 2019: 1-16. https://doi. org/10.1155/2019/9727609

BirdLife International (2008). Birds of Sri Lanka. http://www. ibasrilanka.net/birds.lk/threatened.asp.

Bossuyt, F., M. Meegaskumbura, N. Beenaerts, D.J. Gower, R. Pethiyagoda, K. Roelants, A. Mannaert, M. Wilkinson, M.M. Bahir, K.N.G.P.K.L. Manamendra-Arachchi, C.J. Schneider, O.V. Oommen \& M.C. Milinkovitch (2004). Local endemism within the western Ghats-Sri Lanka biodiversity hotspot. Science 306: 479-481.

Chandrasiri, S.P., C. Dharmarathne \& D. Mahaulpatha (2018). Diversity and Distribution of Avifauna at the Tropical Montane Cloud Forests of Horton Plains National Park. Journal of Tropical Forestry and Environment 8(1): 39-52

Chao, A., R.L. Chazdon, R.K. Colwell \& T.J. Shen (2005). A new statistical approach for assessing similarity of species composition with incidence and abundance data. Ecology Letters 8: 148-159.

Colwell, R.K. \& J.A. Coddington (1994). Estimating terrestrial biodiversity through extrapolation. Philosophical Transactions of the Royal Society of London Series B Biological Sciences 345(1311): 101-118.

Colwell, R.K., A. Chao, N.J. Gotelli, S.Y. Lin, C.X. Mao, R.L. Chazdon \& J.T. Longino (2012). Models and estimators linking individual-based and sample-based rarefaction, extrapolation, and comparison of assemblages. Journal of Plant Ecology 5: 3-21.

De Silva Wijerathne, G. (2014). A Naturalist's Guide to the Birds of Sri Lanka. Oxford John Beaufoy Publishing, Oxford, 176pp.

Ellepola, G. (2014). A study of bird abundance and diversity in Udawattekele forest reserve, Kandy, Sri Lanka. Tigerpaper 41(2): 7-11.

Fernando, C.H. (1990). Zoological survey of Sri Lanka: Freshwater fauna and Fisheries of Sri Lanka. Natural Resources, Energy and Science Authority, Colombo, Sri Lanka.

Gabadage, D.E., W.M.S. Botejue, T.D. Surasinghe, M.M. Bahir, M.B. Madawala, B. Dayananda, V.U. Weeratunga \& D.M.S.S. Karunarathna (2015). Avifaunal diversity in the peripheral areas of the Maduruoya National Park in Sri Lanka: With conservation and management implications. Journal of Asia-Pacific Biodiversity 8(2): 121-132.

Gunatilleke, N., R. Pethiyagoda \& S. Gunatilleke (2008). Biodiversity of Sri Lanka. Journal of National Science Foundation Sri Lanka 36(25): 25-61.

Gunawardena, K. \& D.K. Weerakoon (2012). The Taxonomy and Conservation Status of Birds in Sri Lanka. Ministry of Environment, Colombo, Sri Lanka. 
Harrison, J. (2011). A Field Guide to the Birds of Sri Lanka. $2^{\text {nd }}$ Edition, Oxford University Press, Oxford, 256pp.

Harrison, J. \& T. Worfolk (1999). Field Guide to the Birds of Sri Lanka. Oxford University Press, Oxford, UK, 219pp.

Henry, G.M. (1971). A Guide to the Birds of Ceylon. Oxford University Press, Oxford, UK.

Hettiarachchi, T. \& C.S. Wijesundara (2017). Conservational significance of Dunumadalawa Forest Reserve in Central Sri Lanka based on the endemism of its avifauna. Ceylon Journal of Science 46(3): 21-30.

Karunarathna, D.M.S.S., A.A.T. Amarasinghe \& I.N. Bandara (2011). A survey of the avifaunal diversity of Samanala Nature Reserve, Sri Lanka. BirdingASIA 15: 84-91.

Karunaratna, N. (1986). Udavattekale: The Forbidden Forest of the Kings of Kandy. Department of National Archives, Colombo,



Kotagama, S.W. (1993). Wildlife conservation and development of the south east dry zone. The Southeast dry Zone of Sri Lanka. Agrarian Research and Training Institute, Colombo.

Kotagama, S.W. (2000). How many endemic birds? Loris 22(3): 23-24.

Kotagama, S. \& A. Wijayasingha (1998). SirilakaKurullo (in Sinhala) The Wildlife Heritage Trust of Sri Lanka, Colombo.

Kumara, H.I.G.C. \& V.A.M.P.K. Samarawikrama (2018). Diversity of dragonfly species in the Hakkinda islands of Mahaweliriver in the Gatabe area. Journal of Social Sciences and Humanities Review 3(2): 1-14.

Linder, H. (1995). Setting conservation priorities: the importance of endemism and phylogeny in the southern African orchid genus Herschelia. Conservation Biology 9: 585-595.

Magurran, A.E. (1988). Ecological Diversity and its Measurement. Croom Helm Limited, London, 179pp.

MOE (2012). The National Red List 2012 of Sri Lanka; Conservation Status of the Fauna and Flora. Ministry of Environment, Colombo, Sri Lanka, viii+476pp.

MoMD\&E (2019). Biodiversity Profile - Sri Lanka, Sixth National Report to the Convention on Biological Diversity. Biodiversity Secretariat, Ministry of Mahaweli Development and Environment, Sri Lanka, $211 \mathrm{pp}$

R Core Team (2014). A language and environment for statistical computing. R foundation for statistical computing, Vienna, Austria.

Rasmussen, P.C. \& J.C. Anderton (2005). Birds of South Asia. The Ripley Guide, Vols. 1 and 2. Smithsonian Institution and Lynx Edition, Washington, D.C. and Barcelona, 1,067 pp, 180 pls.
Rathnayake, D.G.R.M.M.K., I.S.I. Arachchi, B.S. Pathirana \& S. Wickramasinghe (2016). The Significance of Habitat Characteristics to the Spatial Distribution of Local Avian Assemblage at Gannoruwa Mountain Forest Reserve, Kandy, Sri Lanka. International Journal of Biodiversity 2016: 1-6. https://doi.org/10.1155/2016/8148910

Samarawickrama, V.A.M.P.K., D.R.N.S. Samarawickrama \& J. Gunawardene (2012). A new site record of Gadaya (Labeo fisheri) in Mahaweli Ganga at Hakkinda, Warathenna, Central Province, Sri Lanka. In: Proceedings of the Eighteenth Sessions of the Sri Lanka Association for Fisheries and Aquatic Resources. National Aquatic Resources Research and Development Agency, Colombo, Sri Lanka, $60 \mathrm{pp}$.

Santiapillai, C. \& C. Wijesundara (2002). An Illustrated Guide to the Endemic Birds of Sri Lanka. Science Education Unit, University of Peradeniya, Sri Lanka.

Silva, I.P., M.C. Prabhath \& A.R. Sumanarathne (2017). Species diversity and abundance of avifauna in and around an urbanized lake situated at Peralanda -Ragama, North Colombo - Sri Lanka. WILDLANKA 5(2): 36-45.

Sinha, A., H. Hariharan, B.S. Adhikari \& R. Krishnamurthy (2019). Bird diversity along riverine areas in the Bhagirathi Valley, Uttarakhand, India. Biodiversity Data Journal 7(e31588): 1-30.

Sri Lanka (2017). The National Environmental Act, 1980. Government Gazette Extraordinary No. 2024/6. June 19, 2017.

Subasinghe, K. \& A.P. Sumanapala (2014). Biological and functional diversity of bird communities in natural and human modified habitats in Northern Flank of Knuckles Mountain Forest Range, Sri Lanka. BIODIVERSITAS 15: 200-205.

Thilakarathne, D., G.N. Hirimuthugoda, T. Lakkana \& S. Kumburegama (2019). Ichthyofauna at Warathenna-Hakkinda environmental protection area in Kandy, Sri Lanka. WILDLANKA 7(4): 181-196.

Warakagoda, D., C. Inskipp, T. Inskipp \& R. Grimmett (2012). Birds of Sri Lanka-Helm Field Guide. Christopher Helm, London, 224pp.

Weerakoon, W.M.B.M.B. (2015). Avifaunal diversity of Udawattakele, an urban forest reserve in the Kandy District. Sciscitator 2: 19-21.

Wijesundara, C. \& C. Santiapillai (2001). The Diversity of resident birds in the campus of the University of Peradeniya, Sri Lanka. Journal of Eastern University Sri Lanka 2(1): 69-82.

Wijesundara, C. \& M.D. Silva (2005). Species diversity, abundance and some aspects of the ecology of birds in selected habitats in the hill region of Sri Lanka. Ceylon Journal of Science 33: 15-31. 

Dr. Albert G. Orr, Griffith University, Nathan, Australia

Dr. Sameer Padhye, Katholieke Universiteit Leuven, Belgium

Dr. Nancy van der Poorten, Toronto, Canada

Dr. Kareen Schnabel, NIWA, Wellington, New Zealand

Dr. R.M. Sharma, (Retd.) Scientist, Zoological Survey of India, Pune, India

Dr. Manju Siliwal, WILD, Coimbatore, Tamil Nadu, India

Dr. G.P. Sinha, Botanical Survey of India, Allahabad, India

Dr. K.A. Subramanian, Zoological Survey of India, New Alipore, Kolkata, India

Dr. P.M. Sureshan, Zoological Survey of India, Kozhikode, Kerala, India

Dr. R. Varatharajan, Manipur University, Imphal, Manipur, India

Dr. Eduard Vives, Museu de Ciències Naturals de Barcelona, Terrassa, Spain

Dr. James Young, Hong Kong Lepidopterists' Society, Hong Kong

Dr. R. Sundararaj, Institute of Wood Science \& Technology, Bengaluru, India

Dr. M. Nithyanandan, Environmental Department, La Ala Al Kuwait Real Estate. Co. K.S.C., Kuwait

Dr. Himender Bharti, Punjabi University, Punjab, India

Mr. Purnendu Roy, London, UK

Dr. Saito Motoki, The Butterfly Society of Japan, Tokyo, Japan

Dr. Sanjay Sondhi, TITLI TRUST, Kalpavriksh, Dehradun, India

Dr. Nguyen Thi Phuong Lien, Vietnam Academy of Science and Technology, Hanoi, Vietnam

Dr. Nitin Kulkarni, Tropical Research Institute, Jabalpur, India

Dr. Robin Wen Jiang Ngiam, National Parks Board, Singapore

Dr. Lional Monod, Natural History Museum of Geneva, Genève, Switzerland.

Dr. Asheesh Shivam, Nehru Gram Bharti University, Allahabad, India

Dr. Rosana Moreira da Rocha, Universidade Federal do Paraná, Curitiba, Brasil

Dr. Kurt R. Arnold, North Dakota State University, Saxony, Germany

Dr. James M. Carpenter, American Museum of Natural History, New York, USA

Dr. David M. Claborn, Missouri State University, Springfield, USA

Dr. Kareen Schnabel, Marine Biologist, Wellington, New Zealand

Dr. Amazonas Chagas Júnior, Universidade Federal de Mato Grosso, Cuiabá, Brasil

Mr. Monsoon Jyoti Gogoi, Assam University, Silchar, Assam, India

Dr. Heo Chong Chin, Universiti Teknologi MARA (UiTM), Selangor, Malaysia

Dr. R.J. Shiel, University of Adelaide, SA 5005, Australia

Dr. Siddharth Kulkarni, The George Washington University, Washington, USA

Dr. Priyadarsanan Dharma Rajan, ATREE, Bengaluru, India

Dr. Phil Alderslade, CSIRO Marine And Atmospheric Research, Hobart, Australia

Dr. John E.N. Veron, Coral Reef Research, Townsville, Australia

Dr. Daniel Whitmore, State Museum of Natural History Stuttgart, Rosenstein, Germany.

Dr. Yu-Feng Hsu, National Taiwan Normal University, Taipei City, Taiwan

Dr. Keith V. Wolfe, Antioch, California, USA

Dr. Siddharth Kulkarni, The Hormiga Lab, The George Washington University, Washington,

D.C., USA

Dr. Tomas Ditrich, Faculty of Education, University of South Bohemia in Ceske

Budejovice, Czech Republic

Dr. Mihaly Foldvari, Natural History Museum, University of Oslo, Norway

Dr. V.P. Uniyal, Wildlife Institute of India, Dehradun, Uttarakhand 248001, India

Dr. John T.D. Caleb, Zoological Survey of India, Kolkata, West Bengal, India

Dr. Priyadarsanan Dharma Rajan, Ashoka Trust for Research in Ecology and the Environment (ATREE), Royal Enclave, Bangalore, Karnataka, India

\section{Fishes}

Dr. Neelesh Dahanukar, IISER, Pune, Maharashtra, India

Dr. Topiltzin Contreras MacBeath, Universidad Autónoma del estado de Morelos, México

Dr. Heok Hee Ng, National University of Singapore, Science Drive, Singapore

Dr. Rajeev Raghavan, St. Albert's College, Kochi, Kerala, India

Dr. Robert D. Sluka, Chiltern Gateway Project, A Rocha UK, Southall, Middlesex, UK

Dr. E. Vivekanandan, Central Marine Fisheries Research Institute, Chennai, India

Dr. Davor Zanella, University of Zagreb, Zagreb, Croatia

Dr. A. Biju Kumar, University of Kerala, Thiruvananthapuram, Kerala, India

Dr. Akhilesh K.V., ICAR-Central Marine Fisheries Research Institute, Mumbai Research

Centre, Mumbai, Maharashtra, India

Dr. J.A. Johnson, Wildlife Institute of India, Dehradun, Uttarakhand, India

\section{Amphibians}

Dr. Sushil K. Dutta, Indian Institute of Science, Bengaluru, Karnataka, India

Dr. Annemarie Ohler, Muséum national d'Histoire naturelle, Paris, France

\section{Reptiles}

Dr. Gernot Vogel, Heidelberg, Germany

Dr. Raju Vyas, Vadodara, Gujarat, India

Dr. Pritpal S. Soorae, Environment Agency, Abu Dubai, UAE.

Prof. Dr. Wayne J. Fuller, Near East University, Mersin, Turkey

Prof. Chandrashekher U. Rivonker, Goa University, Taleigao Plateau, Goa. India

Dr. S.R. Ganesh, Chennai Snake Park, Chennai, Tamil Nadu, India

Dr. Himansu Sekhar Das, Terrestrial \& Marine Biodiversity, Abu Dhabi, UAE
Birds

Dr. Hem Sagar Baral, Charles Sturt University, NSW Australia

Dr. Chris Bowden, Royal Society for the Protection of Birds, Sandy, UK

Dr. Priya Davidar, Pondicherry University, Kalapet, Puducherry, India

Dr. J.W. Duckworth, IUCN SSC, Bath, UK

Dr. Rajah Jayapal, SACON, Coimbatore, Tamil Nadu, India

Dr. Rajiv S. Kalsi, M.L.N. College, Yamuna Nagar, Haryana, India

Dr. V. Santharam, Rishi Valley Education Centre, Chittoor Dt., Andhra Pradesh, India

Dr. S. Balachandran, Bombay Natural History Society, Mumbai, India

Mr. J. Praveen, Bengaluru, India

Dr. C. Srinivasulu, Osmania University, Hyderabad, India

Dr. K.S. Gopi Sundar, International Crane Foundation, Baraboo, USA

Dr. Gombobaatar Sundev, Professor of Ornithology, Ulaanbaatar, Mongolia

Prof. Reuven Yosef, International Birding \& Research Centre, Eilat, Israel

Dr. Taej Mundkur, Wetlands International, Wageningen, The Netherlands

Dr. Carol Inskipp, Bishop Auckland Co., Durham, UK

Dr. Tim Inskipp, Bishop Auckland Co, Durham, UK

Dr. V. Gokula, National College, Tiruchirappalli, Tamil Nadu, India

Dr. Arkady Lelej, Russian Academy of Sciences, Vladivostok, Russia

Dr. Simon Dowell, Science Director, Chester Zoo, UK

Dr. Mário Gabriel Santiago dos Santos, Universidade de Trás-os-Montes e Alto Douro,

Quinta de Prados, Vila Real, Portugal

Dr. Grant Connette, Smithsonian Institution, Royal, VA, USA

Dr. M. Zafar-ul Islam, Prince Saud Al Faisal Wildlife Research Center, Taif, Saudi Arabia

Mammals

Dr. Giovanni Amori, CNR - Institute of Ecosystem Studies, Rome, Italy

Dr. Anwaruddin Chowdhury, Guwahati, India

Dr. David Mallon, Zoological Society of London, UK

Dr. Shomita Mukherjee, SACON, Coimbatore, Tamil Nadu, India

Dr. Angie Appel, Wild Cat Network, Germany

Dr. P.O. Nameer, Kerala Agricultural University, Thrissur, Kerala, India

Dr. Ian Redmond, UNEP Convention on Migratory Species, Lansdown, UK

Dr. Heidi S. Riddle, Riddle's Elephant and Wildlife Sanctuary, Arkansas, USA

Dr. Karin Schwartz, George Mason University, Fairfax, Virginia.

Dr. Lala A.K. Singh, Bhubaneswar, Orissa, India

Dr. Mewa Singh, Mysore University, Mysore, India

Dr. Paul Racey, University of Exeter, Devon, UK

Dr. Honnavalli N. Kumara, SACON, Anaikatty P.O., Coimbatore, Tamil Nadu, India

Dr. Nishith Dharaiya, HNG University, Patan, Gujarat, India

Dr. Spartaco Gippoliti, Socio Onorario Società Italiana per la Storia della Fauna "Giuseppe

Altobello", Rome, Italy

Dr. Justus Joshua, Green Future Foundation, Tiruchirapalli, Tamil Nadu, India

Dr. H. Raghuram, The American College, Madurai, Tamil Nadu, India

Dr. Paul Bates, Harison Institute, Kent, UK

Dr. Jim Sanderson, Small Wild Cat Conservation Foundation, Hartford, USA

Dr. Dan Challender, University of Kent, Canterbury, UK

Dr. David Mallon, Manchester Metropolitan University, Derbyshire, UK

Dr. Brian L. Cypher, California State University-Stanislaus, Bakersfield, CA

Dr. S.S. Talmale, Zoological Survey of India, Pune, Maharashtra, India

Prof. Karan Bahadur Shah, Budhanilakantha Municipality, Kathmandu, Nepal

Dr. Susan Cheyne, Borneo Nature Foundation International, Palangkaraja, Indonesia

Dr. Hemanta Kafley, Wildlife Sciences, Tarleton State University, Texas, USA

\section{Other Disciplines}

Dr. Aniruddha Belsare, Columbia MO 65203, USA (Veterinary)

Dr. Mandar S. Paingankar, University of Pune, Pune, Maharashtra, India (Molecular)

Dr. Jack Tordoff, Critical Ecosystem Partnership Fund, Arlington, USA (Communities)

Dr. Ulrike Streicher, University of Oregon, Eugene, USA (Veterinary)

Dr. Hari Balasubramanian, EcoAdvisors, Nova Scotia, Canada (Communities)

Dr. Rayanna Hellem Santos Bezerra, Universidade Federal de Sergipe, São Cristóvão, Brazil

Dr. Jamie R. Wood, Landcare Research, Canterbury, New Zealand

Dr. Wendy Collinson-Jonker, Endangered Wildlife Trust, Gauteng, South Africa

Dr. Rajeshkumar G. Jani, Anand Agricultural University, Anand, Gujarat, India

Dr. O.N. Tiwari, Senior Scientist, ICAR-Indian Agricultural Research Institute (IARI), New

Delhi, India

Dr. L.D. Singla, Guru Angad Dev Veterinary and Animal Sciences University, Ludhiana, India

Dr. Rupika S. Rajakaruna, University of Peradeniya, Peradeniya, Sri Lanka

Dr. Bahar Baviskar, Wild-CER, Nagpur, Maharashtra 440013, India

Reviewers 2018-2020

Due to pausity of space, the list of reviewers for 2018-2020 is available online.
The opinions expressed by the authors do not reflect the views of the Journal of Threatened Taxa, Wildlife Information Liaison Development Society, Zoo Outreach Organization, or any of the partners. The journal, the publisher, the host, and the partners are not responsible for the accuracy of the political boundaries shown in the maps by the authors.

\footnotetext{
Print copies of the Journal are available at cost. Write to:

The Managing Editor, JoTT,

c/o Wildlife Information Liaison Development Society,

No. 12, Thiruvannamalai Nagar, Saravanampatti - Kalapatti Road,

Saravanampatti, Coimbatore, Tamil Nadu 641035, India

ravi@threatenedtaxa.org
} 


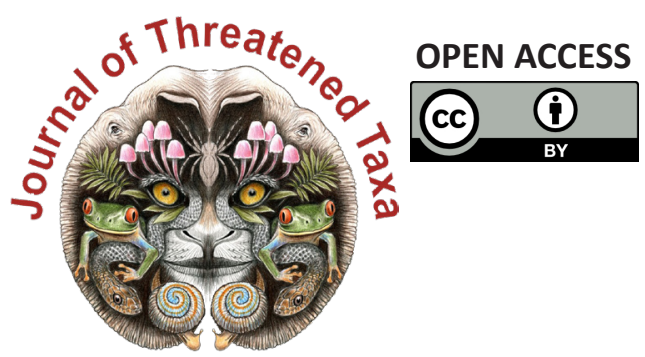

www.threatenedtaxa.org

The Journal of Threatened Taxa (JoTT) is dedicated to building evidence for conservation globally by publishing peer-reviewed articles online every month at a reasonably rapid rate at www.threatenedtaxa.org. All articles published in JoTT are registered under Creative Commons Attribution 4.0 International License unless otherwise mentioned. JoTT allows allows unrestricted use, reproduction, and distribution of articles in any medium by providing adequate credit to the author(s) and the source of publication.

\section{ISSN $0974-7907$ (Online) | ISSN $0974-7893$ (Print)}

\section{October 2021 | Vol. 13 | No. 12 | Pages: 19675-19886 \\ Date of Publication: 26 October 2021 (Online \& Print) DOI: 10.11609/jott.2021.13.12.19675-19886}

Articles

Roosting habits and habitats of the Indian Flying Fox Pteropus medius Temminck, 1825 in the northern districts of Tamil Nadu, India

- M. Pandian \& S. Suresh, Pp. 19675-19688

Diversity and distribution of avifauna at Warathenna-Hakkinda Environmental Protection Area in Kandy, Sri Lanka

- Dinelka Thilakarathne, Tithira Lakkana, Gayan Hirimuthugoda, Chaminda Wijesundara \& Shalika Kumburegama, Pp. 19689-19701

Grass species composition in tropical forest of southern India

- M. Ashokkumar, S. Swaminathan \& R. Nagarajan, Pp. 19702-19713

\section{Communications}

Habitat use and conservation threats to Wild Water Buffalo Bubalus arnee (Mammalia: Artiodactyla: Bovidae) in Koshi Tappu Wildlife Reserve, Nepal

- Reeta Khulal, Bijaya Neupane, Bijaya Dhami, Siddhartha Regmi, Ganesh Prasad Tiwari \& Manita Parajuli, Pp. 19714-19724

Get my head around owls: people perception and knowledge about owls of Andaman Islands

- Shanmugavel Sureshmarimuthu, Santhanakrishnan Babu, Nagaraj Rajeshkumar \& Honnavalli Nagaraj Kumara, Pp. 19725-19732

Abundance and diversity of threatened birds in Nangal Wetland, Punjab, India - Rajwinder Kaur \& Onkar Singh Brraich, Pp. 19733-19742

Evaluation of fish diversity and abundance in the Kabul River with comparisons between reaches above and below Kabul City, Afghanistan

- Ugyen Kelzang, Ahmad Farid Habibi \& Ryan J. Thoni, Pp. 19743-19752

New record of Myrmarachne melanocephala MacLeay, 1839 (Araneae: Salticidae) from Jharkhand, India and biogeographical implications of the co-occurrence of its ant model Tetraponera rufonigra Jerdon, 1851

- Rahul Kumar, Mirtunjay Sharma \& Ajay Kumar Sharma, Pp. 19753-19761

Diversity of spiders (Arachnida: Araneae) and the impact of pruning in Indian sandalwood plantations from Karnataka, India

-S. Padma 1 \& R. Sundararaj, Pp. 19762-19772

New records of cheilostome Bryozoa from the eastern coast of India encrusting on the exoskeleton of live horseshoe crabs of Indian Sundarbans

- Swati Das, Maria Susan Sanjay, Basudev Tripathy, C. Venkatraman \& K.A. Subramanian, Pp. 19773-19780

On the pteridophytes of Bherjan-Borajan-Padumoni Wildlife Sanctuary, Assam, India - Pranjal Borah \& Jayanta Barukial, Pp. 19781-19790

Population status of Heritiera fomes Buch.-Ham., a threatened species from Mahanadi Mangrove Wetland, India

- Sudam Charan Sahu, Manas Ranjan Mohanta \& N.H. Ravindranath, Pp. 19791-19798

Additions to the lichenized and lichenicolous fungi of Jammu \& Kashmir from Kishtwar High Altitude National Park

- Vishal Kumar, Yash Pal Sharma, Siljo Joseph, Roshinikumar Ngangom \& Sanjeeva Nayaka, Pp. 19799-19807

\section{Short Communications}

Is release of rehabilitated wildlife with embedded lead ammunition advisable? Plumbism in a Jaguar Panthera Onca (Mammalia: Carnivora: Felidae), survivor of gunshot wounds - Eduardo A. Díaz, Carolina Sáenz, E. Santiago Jiménez, David A. Egas \& Kelly Swing, Pp. 19808-19812

New record of the Sewing Needle Zipper Loach Paracanthocobitis linypha Singer \& Page, 2015 (Teleostei: Cypriniformes: Nemacheilidae) from the Chindwin drainage of Manipur, India

- Yumnam Rameshori, Yengkhom Chinglemba \& Waikhom Vishwanath, Pp. 19813-19817

Field identification characters to diagnose Microhyla mukhlesuri from closely related M. mymensinghensis (Amphibia: Microhylidae) and range extension of $M$. mukhlesuri up to West Bengal State, India

- Suman Pratihar \& Kaushik Deuti, Pp. 19818-19823
First report of Scipinia horrida (Stål) (Heteroptera: Reduviidae) from Assam, with comments on related genus Irantha Stål

- Anjana Singha Naorem, Santana Saikia, Anandita Buragohain, Rubina Azmeera Begum, Swapnil S. Boyane \& Hemant V. Ghate, Pp. 19824-19830

Flesh fly (Diptera: Sarcophagidae): male terminalia, diversity and expanded geographical distribution from India

- Kanholi Sreejith, Shuvra Kanti Sinha, Santanu Mahato \& Edamana Pushpalatha, Pp. 1983119836

Checklist of moths (Heterocera) of Tadong, Sikkim, India

- Prayash Chettri, Yuki Matsui, Hideshi Naka \& Archana Tiwari, Pp. 19837-19848

New distribution records of Begonia L., B. murina Craib and B. poilanei Kiew (Begoniaceae: Cucurbitales) for Laos

- Phongphayboun Phonepaseuth, Phetlasy Souladeth, Soulivanh Lanorsavanh, Shuichiro Tagane, Thyraphon Vongthavone \& Keooudone Souvannakhoummane Pp. 19849-19854

Notes

A recent sighting of the Stripe-backed Weasel Mustela strigidorsa (Mammalia: Carnivora: Mustelidae) in Hkakabo Razi Landscape, Myanmar

- Sai Sein Lin Oo, Tun Tun, Kyaw Myo Naing \& Paul Jeremy James Bates, Pp. 19855-19859

Are the uplifted reef beds in North Andaman letting nesting Olive Ridley Sea Turtle Lepidochelys olivacea stranded?

- Nehru Prabakaran, Anoop Raj Singh \& Vedagiri Thirumurugan, Pp. 19860-19863

First record of the orb-weaving spider Araneus tubabdominus Zhu \& Zhang, 1993 (Araneae: Araneidae) from India

- Souvik Sen, John T.D. Caleb \& Shelley Acharya, Pp. 19864-19866

The genus Catapiestus Perty, 1831 (Coleoptera: Tenebrionidae: Cnodalonini) from Arunachal Pradesh with one new record to India

- V.D. Hegde \& Sarita Yadav, Pp. 19867-19869

Rediscovery and extended distribution of Indigofera santapaui Sanjappa (Leguminosae: Papilionoideae) from the states of Maharashtra and Gujarat, India

- Kumar Vinod Chhotupuri Gosavi, Sanjay Gajanan Auti, Sharad Suresh Kambale \& Munivenkatappa Sanjappa, Pp. 19870-19873

Additional distribution records of Ceropegia anjanerica, an endemic and 'Endangered' lantern flower of the northern Western Ghats, India

- Samir Shrikant Maity, Ajay Natha Gangurde, Sharad Suresh Kambale, Avinash Ramchandra Gholave, Avinash Asraji Adsul, Ganesh Babaso Pawar \& Kumar Vinod Chhotupuri Gosavi, Pp. 19874-19877

Notes on the extended distribution of Impatiens megamalayana, a recently described balsam in Western Ghats, India

- Anoop P. Balan \& A.J. Robi, Pp. 19878-19883

Book Review

A look over on the scented tree of India (Santalum album - S. Suresh Ramanan \& A. Arunachalam, Pp. 19884-19886
Publisher \& Host
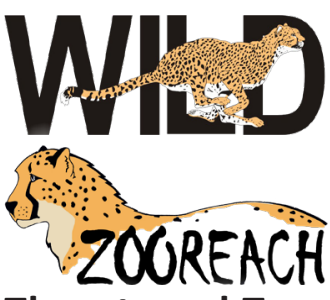

Threatened Taxa 